\title{
Data Abstraction: A General Framework to Handle Program Verification of Data Structures
}

\author{
Julien Braine ${ }^{1,3}$, Laure Gonnord ${ }^{2,3}[0000-0002-8013-1611] \star$, and David \\ Monniaux ${ }^{4}[0000-0001-7671-6126]$ \\ 1 ENS de Lyon \\ Julien.Braine@ens-lyon.fr \\ 2 Université Claude Bernard Lyon 1 \\ Laure.Gonnord@ens-lyon.fr \\ 3 LIP (CNRS, ENS de Lyon, Inria, Univ. Lyon), Lyon, France \\ 4 Univ. Grenoble Alpes, CNRS, Grenoble INP $\star \star \star$, VERIMAG, Grenoble, France \\ David.Monniaux@univ-grenoble-alpes.fr
}

\begin{abstract}
Proving properties on programs accessing data structures such as arrays often requires universally quantified invariants, e.g., "all elements below index $i$ are nonzero". In this article, we propose a general data abstraction scheme operating on Horn formulas, into which we recast previously published abstractions. We show that our instantiation scheme is relatively complete: the generated purely scalar Horn clauses have a solution (inductive invariants) if and only if the original problem has one expressible by the abstraction.
\end{abstract}

Keywords: Static analysis · Horn clauses · Abstraction
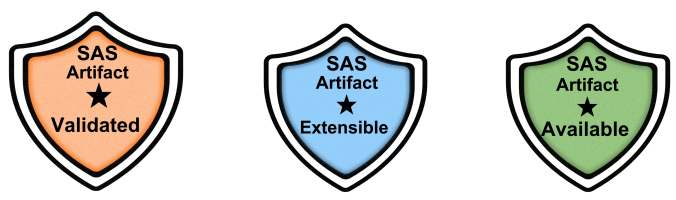

\section{Introduction}

Static analysis of programs containing unbounded data structures is challenging, as most interesting properties require quantifiers. Even stating that all elements of an array are equal to 0 requires them $(\forall i a[i]=0)$, let alone more complex cases such as Example 1. In general, the satisfiability of arbitrary quantified properties on unbounded data structures is undecidable [3], thus there is no algorithm for checking that such properties are inductive, nor inferring them.

$\star \star \star$ Institute of Engineering Univ. Grenoble Alpes

* This work was partially funded by the French National Agency of Research in the CODAS Project (ANR-17-CE23-0004-01) 
The first step is to select an abstract domain to search for invariants, e.g., properties of the form $\forall i, P(a[i])$ for some predicate $P$ and array $a$. In this paper, we describe a transformation from and to Horn clauses such that these properties may be expressed in the transformed Horn clauses without using quantifiers. For the array data structure, our sheme can optionally completely remove arrays from the transformed Horn clauses. This transformation is sound and relatively complete: the resulting problem, to be fed to a solver for Horn clauses, such as Eldarica or Z3, has a solution if and only if the original one has one within the chosen abstract domain. In short, we reduce problems involving quantifiers and arrays to problems that do not, with no loss of precision with respect to the abstract domain.

Example 1 (Running example). The following program initializes an array to even values, then increases all values by one and checks that all values are odd. We wish to prove that the assertion is verified.

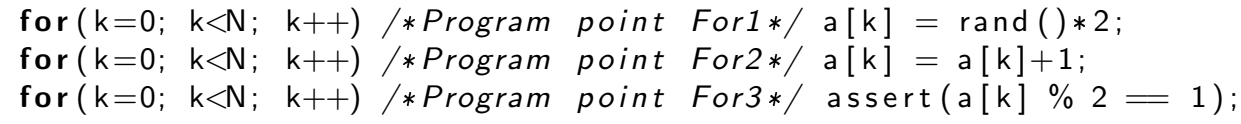

Contributions (i) an abstraction framework for Horn clauses using unbounded data structures, that we call data abstraction; (ii) the analysis of a property we call relative completeness in this framework (iii) and the use of that framework to handle programs with arrays and its experimental evaluation.

Contents Section 2 introduces Horn Clauses' concepts and notations. Sections 3 and 4 expose our data abstraction framework and its relative completeness analysis. Section 5 considers a data abstraction for arrays. Finally, Section 6 proposes a full algorithm to analyze programs with arrays and its experimental evaluation. The appendix contains the proofs of theorems.

\section{Preliminaries: Horn clauses}

\subsection{Solving Programs with assertions using Horn Clauses}

Programs with assertions can be transformed into Horn clauses using tools such as SeaHorn 8 or JayHorn 13. The syntax of Horn clauses is recalled in Def. 2 . A basic transformation consists in associating a predicate to each point of the control flow graph ; control edges are inductive relations (clauses), and assertions $A$ are clauses $\neg A \rightarrow$ false $5^{5}$

\footnotetext{
5 Tools such as SeaHorn 8 handle richer languages such as LLVM bytecode, but the generated clauses are more complex and further removed from their initial semantics. Such clauses fall within our data abstraction framework, but not within the scope of the experimental evaluation of this paper.
} 
Example 2 (Example 1 transformed into Horn clauses). All predicates For $_{i}$ have arity 3 (1 array and 2 integer parameters) and Clause (4) in bold, will be used throughout the paper.

$$
\begin{array}{r}
\text { For } 1(a, N, 0) \\
\text { For } 1(a, N, k) \wedge k<N \rightarrow \text { For } 1(a[k \leftarrow r * 2], N, k+1) \\
\operatorname{For} 1(a, N, k) \wedge k \geq N \rightarrow \text { For } 2(a, N, 0) \\
\operatorname{For2}(\boldsymbol{a}, \boldsymbol{N}, \boldsymbol{k}) \wedge \boldsymbol{k}<N \rightarrow \boldsymbol{F o r} \mathbf{2}(\boldsymbol{a}[\boldsymbol{k} \leftarrow \boldsymbol{a}[\boldsymbol{k}]+\mathbf{1}], \boldsymbol{N}, \boldsymbol{k}+\mathbf{1}) \\
\operatorname{For} 2(a, N, k) \wedge k \geq N \rightarrow \text { For } 3(a, N, 0) \\
\text { For } 3(a, N, k) \wedge k<N \wedge a[k] \% 2 \neq 1 \rightarrow \text { false } \\
\text { For } 3(a, N, k) \wedge k<N \rightarrow \text { For } 3(a, N, k+1)
\end{array}
$$

Variables are local: the $a$ of Clause 1 is not formally related to the $a$ of Clause 4

A solution to such a system of Horn clauses is a set of inductive invariants suitable for proving the desired properties. Horn clause systems can be solved by tools such as Z3, Eldarica, .... A "Sat" answer means that the inductive invariants exist and thus the program is correct ${ }^{6}$ "Unsat" means that a counterexample was found, leading to an assertion violation. "Unknown" means the tool fails to converge on a possible invariant. Finally, the tool may also timeout.

\subsection{Horn Clauses and Horn problems}

In our setting, a Horn clause is a boolean expression over free variables and predicates with at most one positive predicate.

Definition 1 (Expressions expr, positive and negative predicates $P$, models $\mathcal{M}$ and semantics expr(vars), $\left.\llbracket \operatorname{expr} \rrbracket_{\mathcal{M}}\right)$. In this paper we do not constrain the theory on which expressions are written, the only constraint being that the backend solver must handle it. An expression may contain quantifiers, free variables and predicates. A predicate is a typed name which will be a set when interpreted in a model.

Expression evaluation: There are two evaluation contexts for expressions:

1. Models, written $\mathcal{M}$ : map each predicate to a set of the corresponding domain

2. Environments, written vars, that to each free variable of the expression associates a value of the corresponding type's domain

$\llbracket$ expr $\rrbracket_{\mathcal{M}}$ denotes the evaluation of an expression expr in a model $\mathcal{M}$, expr (vars) denotes its the evaluation in the environment vars, $\llbracket \operatorname{expr}($ vars $) \rrbracket_{\mathcal{M}}$ denotes joint evaluation. Furthermore, if an expression value is independent of the model or environment, we may use it directly as its evaluation.

Positive and negative predicates : A predicate instance in a boolean expression expr is deemed negative (resp. positive) if and only if there is a negation (resp. no negation) in front of it when expr is in negative normal form.

\footnotetext{
${ }^{6} \mathrm{Z} 3$ is both a SMT solver and a tool for solving Horn clauses. In SMT, a "Sat" answer often means a counterexample trace invalidating a safety property. In contrast, in Horn solving, "Sat" means a safety property is proved.
} 
Definition 2 (Horn Clauses, extended, normalized, satisfiability). A Horn clause is simply any expression without quantifiers (but with free variables) containing at most one positive predicate.

Extended Horn clause : a Horn clause which may use quantifiers.

Normalized Horn clause : Normalized Horn clauses are in the form $P_{1}\left(e_{1}\right) \wedge$ $\ldots \wedge P_{n}\left(e_{n}\right) \wedge \phi \rightarrow P^{\prime}\left(e^{\prime}\right)$ where:

$-e_{1}, \ldots, e_{n}, \phi, e^{\prime}$ are expressions without predicates but with free variables.

$-P_{1}, \ldots, P_{n}$ are the "negative" predicates

- $P^{\prime}$ is the positive predicate or some expression

Satisfiability: A set of Horn clauses $\mathfrak{C}$ is said to be satisfiable if and only if $\exists \mathcal{M}, \forall C \in \mathfrak{C}, \llbracket \forall$ vars,$C($ vars $) \rrbracket_{\mathcal{M}}$.

In this paper, we will denote clauses in capital letters: $C$, sets of clauses in Fraktur: $\mathfrak{C}$, and models in calligraphic: $\mathcal{M}$.

Definition 3 (Notations ite, $f[a \leftarrow b]$ ).

For a boolean expression $b$ and expressions $e_{1}, e_{2}$, we define the expression "if-then-else", written ite $\left(b, e_{1}, e_{2}\right)$, evaluating to $e_{1}$ when $b$ and to $e_{2}$ when $\neg b$.

For a function $f$ (i.e. an environment) or an array, we define $f[a \leftarrow b]$ as $f[a \leftarrow b](x)=i t e(x=a, b, f(x))$

Example 3 (Satisfiability of Example 2). The following model satisfies Example2 with $(a, N, k) \in \mathbb{N}^{3}$.

1. $\mathcal{M}($ For 1$)=\{(a, N, k) \mid k<N \wedge \forall i<k, a[i] \% 2=0\}$

2. $\mathcal{M}($ For 2$)=\{(a, N, k) \mid k<N \wedge \forall i<k, a[i] \% 2=1 \wedge \forall k \leq i<N, a[i] \% 2=0\}$

3. $\mathcal{M}(F o r 3)=\{(a, N, k) \mid k<N \wedge \forall i<N, a[i] \% 2=1\}$

Horn clauses constrain models in two ways: those with a positive predicate force the model to be a post-fixpoint of an induction relation; those without are assertions that force the model to not contain elements violating the assertion.

Horn clauses are the syntactic objects we use to write Horn problems. Theorem 2 formalizes the link between Horn problems and Horn clauses.

Definition 4 (Horn Problem $H$, defines $\left.f_{H}, \mathcal{U}_{H}\right)$. A Horn problem $H$ is a pair $\left(f_{H}, \mathcal{U}_{H}\right)$ where (i) $f_{H}$ is a monotone function over models with order $\mathcal{M}_{1} \leq \mathcal{M}_{2} \equiv \forall P, \mathcal{M}_{1}(P) \subseteq \mathcal{M}_{2}(P)$. (ii) $\mathcal{U}_{H}$ is a model. It is said to be satisfiable if and only if lfp $f_{H} \leq \mathcal{U}_{H}$ (where lfp is the least fixpoint operator).

Theorem 1 (Horn problems as a condition on models, defines $H(\mathcal{M})$ ). A Horn problem $H$ is satisfiable if and only if $\exists \mathcal{M}, f_{H}(\mathcal{M}) \leq \mathcal{M} \wedge \mathcal{M} \leq \mathcal{U}_{H}$, also written $\exists \mathcal{M}, H(\mathcal{M})$ with $H(\mathcal{M})={ }_{\text {def }} f_{H}(\mathcal{M}) \leq \mathcal{M} \wedge \mathcal{M} \leq \mathcal{U}_{H}$.

Theorem 2 (Horn clauses as Horn problems, defines $H_{\mathfrak{C}}$ ).

Let $\mathfrak{C}$ be a set of Horn clauses. There exists a Horn problem $H_{\mathfrak{C}}$, such that for any model $\mathcal{M}, H_{\mathfrak{C}}(\mathcal{M})=\forall C \in \mathfrak{C}, \llbracket \forall$ vars,$C($ vars $) \rrbracket_{\mathcal{M}}$. Thus, satisfiable $(\mathfrak{C}) \equiv$ $\operatorname{satisfiable}\left(H_{\mathfrak{C}}\right)$. 


\subsection{Horn problem induced by an abstraction}

Static analysis by abstract interpretation amounts to searching for invariants (i.e., models of Horn clauses in our setting) within a subset of all possible invariants called an abstract domain; elements of that subset are said to be expressible by the abstraction. We formalize abstraction as a Galois connection [4], that is, a pair $(\alpha, \gamma)$ where $\alpha$ denotes abstraction (i.e. simplification) and $\gamma$ denotes the semantics (i.e. what the abstraction corresponds to) of abstract elements.

Definition 5 (Models expressible by the abstraction $\mathcal{G}$ ). We say that a model $\mathcal{M}$ is expressible by an abstraction $\mathcal{G}$ if and only if $\mathcal{M}=\gamma_{\mathcal{G}} \circ \alpha_{\mathcal{G}}(\mathcal{M})$ or equivalently $\exists \mathcal{M}^{\#}, \mathcal{M}=\gamma_{\mathcal{G}}\left(\mathcal{M}^{\#}\right)$.

Example 4 (Models expressible by an abstraction). Consider the model $\mathcal{M}$ from Example 3. This model is expressible by the abstraction $\mathcal{G}$ such that $\forall P \in$ $\{$ For 1, For 2, For 3$\}$ :

1. $\alpha_{\mathcal{G}}(\mathcal{M})(P)=\{(\boldsymbol{i}, a[i], N, k) \mid(a, N, k) \in \mathcal{M}(P)\}$

2. $\gamma_{\mathcal{G}}\left(\mathcal{M}^{\#}\right)(P)=\left\{(a, N, k) \mid \forall i,(i, a[i], N, k) \in \mathcal{M}^{\#}(P)\right\}$

but not by the abstraction $\mathcal{G}^{\prime}$ such that $\forall P \in\{$ For 1, For 2, For 3$\}$ :

1. $\alpha_{\mathcal{G}^{\prime}}(\mathcal{M})(P)=\{(a[i], N, k) \mid(a, N, k) \in \mathcal{M}(P)\}$

2. $\gamma_{\mathcal{G}^{\prime}}\left(\mathcal{M}^{\#}\right)(P)=\left\{(a, N, k) \mid \forall i,(a[i], N, k) \in \mathcal{M}^{\#}(P)\right\}$

The idea is that the abstraction $\mathcal{G}$ keeps the relationships between indices and values $(i, a[i])$, which is all that is needed for our invariants, whereas $\mathcal{G}^{\prime}$ forgets the indices and only keeps information about the values, which is insufficient. Section 5 details what each abstraction expresses on arrays.

Definition 6 (Abstraction of a Horn problem $a b s(\mathcal{G}, H)$ ). The abstraction of Horn problem $H$ by a Galois connection $\mathcal{G}$ noted abs $(\mathcal{G}, H)$, is defined by (i) $f_{a b s(\mathcal{G}, H)}=\alpha_{\mathcal{G}} \circ f_{H} \circ \gamma_{\mathcal{G}}$ (ii) $\mathcal{U}_{a b s(\mathcal{G}, H)}=\alpha_{\mathcal{G}}\left(\mathcal{U}_{H}\right)$.

Theorem 3 (Definition abs $(\mathcal{G}, H)$ is correct). For all $\mathcal{M}^{\#}$, the following statements are equivalent (with the notation $H(\mathcal{M})$, where $H$ is a Horn problem and $\mathcal{M}$ a possible model, from Theorem 1): (i) $\operatorname{abs}(\mathcal{G}, H)\left(\mathcal{M}^{\#}\right)$ (ii) $H\left(\gamma_{\mathcal{G}}\left(\mathcal{M}^{\#}\right)\right)$ (iii) $\operatorname{abs}(\mathcal{G}, H)\left(\alpha_{\mathcal{G}} \circ \gamma_{\mathcal{G}}\left(\mathcal{M}^{\#}\right)\right)$.

Remark 1. From this theorem, it follows that:

1. $a b s(\mathcal{G}, H)$ corresponds to the desired abstraction as: $H$ is satisfiable by a model expressible by the abstraction $\left(\gamma_{\mathcal{G}}\left(\mathcal{M}^{\#}\right)\right)$ iff $a b s(\mathcal{G}, H)$ is satisfiable.

2. $\operatorname{abs}(\mathcal{G}, H)\left(\mathcal{M}^{\#}\right)$ is constructible from $H$ and $\gamma_{\mathcal{G}}$. This will be used in Th. 5

\subsection{Horn clauses transformations}

A transformation is sound if it never transforms unsatisfiable Horn clauses (incorrect programs) into satisfiable ones (correct programs), complete if it never transforms satisfiable Horn clauses into unsatisfiable ones. A transformation is complete relative to an abstraction if it never transforms Horn clauses satisfiable in the abstract domain into unsatisfiable ones. Together, soundness and relative completeness state that the transformation implements exactly the abstraction. 
Definition 7 (Soundness, Completeness, Relative completeness). A transformation alg from Horn clauses to Horn clauses is said to be:

- sound if and only if $\forall \mathfrak{C}, H_{\text {alg( }(\mathfrak{C})}$ satisfiable $\Rightarrow H_{\mathfrak{C}}$ satisfiable.

- complete if and only if $\forall \mathfrak{C}, H_{\mathfrak{C}}$ satisfiable $\Rightarrow H_{\text {alg }(\mathfrak{C})}$ satisfiable .

- complete relative to $\mathcal{G}$ iff $\forall \mathfrak{C}, a b s\left(\mathcal{G}, H_{\mathfrak{C}}\right)$ satisfiable $\Rightarrow H_{\text {alg( }(\mathfrak{C})}$ satisfiable .

Theorem 4 (Soundness with relative completeness is abs). If a transformation alg is sound and complete relative to $\mathcal{G}$, then $\forall \mathfrak{C}, H_{\text {alg }(\mathfrak{C})}$ satisfiable $\equiv$ $\operatorname{abs}\left(\mathcal{G}, H_{\mathfrak{C}}\right)$ satisfiable.

Relative completeness is rarely ensured in abstract interpretation; examples include some forms of policy iteration, which compute the least inductive invariant in the abstract domain. Widening operators, very widely used, break relative completeness. Previous works on arrays do not analyze relative completeness.

In this paper, we present a framework to define abstractions on data such that the relative completeness of transformations is analyzed, and proved when possible. To do so, our abstraction scheme is divided into two algorithms: (i) one that computes $a b s\left(\mathcal{G}, H_{\mathfrak{C}}\right)$ and thus is sound and complete relative to $\mathcal{G}$ but uses extended Horn clauses (i.e. Horn clauses with additional quantifiers); (ii) another which transforms these extended Horn clauses back into Horn clauses and ensures soundness and strives to ensure completeness - and is shown to ensure it in the setting of our tool. When the second algorithm ensures completeness, the framework provides an abstraction algorithm from Horn clauses to Horn clauses which is both sound and complete relative to the abstraction.

\section{Data Abstraction: Abstracting Horn Clauses}

\subsection{Implementing Horn Clauses Abstraction}

The abstraction on the syntax of Horn clauses is done by choosing a predicate to abstract. This approach can then be successively used on several predicates.

In this paper, we consider a subset of abstractions we call data abstractions. The semantics of a predicate is a set of unknown values and an abstraction is simply a relation in the form of a Galois connection between that set of unknown values to a "simpler" set of unknown values. The key particularity of data abstractions is that the abstraction of this set of unknown values is defined by the abstraction of its individual elements, the "data". This allows us to take advantage of the syntax of Horn clauses because the "data" is simply the expressions passed as parameters to a predicate. Furthermore, as our goal is to syntactically modify the Horn clauses, we require that the abstraction can be encoded by an explicit formula that will be used syntactically during the transformation.

Definition 8 (Data abstraction $\sigma$, defines $F_{\sigma}, \alpha_{\sigma}, \gamma_{\sigma}, \mathcal{G}_{\sigma}^{P}$ ).

Let $\mathscr{C}$ and $\mathcal{A}$ be sets. A data abstraction $\sigma$ is a function from $\mathscr{C}$ to $\mathcal{P}(\mathcal{A})$ and we write $F_{\sigma}$ the formula encoding its inclusion relation : $F_{\sigma}\left(a^{\#}, a\right) \equiv a^{\#} \in \sigma(a)$.

\footnotetext{
${ }^{7}$ Classically, we denote abstract elements $(\in \mathcal{A})$ with sharps $(\#)$.
} 
It defines a Galois connection from $\mathcal{P}(\mathscr{C})$ to $\mathcal{P}(\mathcal{A})$ as follows: for $S \subseteq \mathscr{C}$, $S^{\#} \subseteq \mathcal{A}, \alpha_{\sigma}(S)=\bigcup_{a \in S} \sigma(a)$ and $\gamma_{\sigma}\left(S^{\#}\right)=\left\{a \in \mathscr{C} \mid \sigma(a) \subseteq S^{\#}\right\}$.

This Galois connection can be applied to a predicate $P$, thus yielding the Galois connection $\mathcal{G}_{\sigma}^{P}$ defined by $\alpha_{\mathcal{G}_{\sigma}^{P}}(\mathcal{M})\left(P^{\prime}\right)=$ ite $\left(P^{\prime}=P, \alpha_{\sigma}(\mathcal{M}(P)), \mathcal{M}\left(P^{\prime}\right)\right)$ and $\gamma_{\mathcal{G}_{\sigma}^{P}}\left(\mathcal{M}^{\#}\right)\left(P^{\prime}\right)=\operatorname{ite}\left(P^{\prime}=P, \gamma_{\sigma}\left(\mathcal{M}^{\#}(P)\right), \mathcal{M}^{\#}\left(P^{\prime}\right)\right)$.

Example 5 (Cell $l_{1}$ abstraction of an array).

Cell $l_{1}$ abstracts an array by the set of its cells (i.e. pairs of index and value). $\sigma_{\text {Cell }_{1}}(a)=\{(i, a[i])\} \quad F_{\sigma_{\text {Cell }_{1}}}((i, v), a) \equiv v=a[i]$

Remark 2. This data abstraction $\sigma_{C e l l_{1}}$ essentially abstracts a function $a$ from an arbitrary index type $I$ to an arbitrary value type by its graph $\{(i, a(i)) \mid i \in I\}$. As such, it does not lose information on individual arrays: two functions are identical if and only if they have the same graph (functional extensionality).

However, the associated $\alpha_{\sigma_{\text {Cell }_{1}}}$ is not injective and loses information. This is essentially because when one takes the superposition of the graphs of two or more functions, there is no way to recover which part of the graph corresponds to which function. Consider for example $a_{0}: 0 \mapsto 0,1 \mapsto 1$ and $a_{1}: 0 \mapsto 1,1 \mapsto 0$. Then, $\alpha_{\sigma_{\text {Cell }_{1}}}\left(\left\{a_{0}, a_{1}\right\}\right)=\{0,1\} \times\{0,1\}$; and thus $\alpha_{\sigma_{\text {Cell }_{1}}}\left(\left\{a_{0}, a_{1}\right\}\right)$ contains not only $a_{0}$ and $a_{1}$, but also the constant arrays 0 and 1 .

We now give the syntactic transformation on Horn clauses, so that the Horn problem induced by the transformed clauses corresponds to the abstraction of the desired predicate by the given data abstraction. We rely on Theorem 3 . which states how the abstract Horn problem must be constructed and find its syntactical counterpart. Thus, if $P$ is the predicate to be abstracted by $\sigma, \mathcal{M}(P)$ must be replaced by $\left(\gamma_{\sigma}\left(\mathcal{M}\left(P^{\#}\right)\right)\right)($ expr $)$, where $\mathcal{M}\left(P^{\#}\right)$ is the abstracted set and $P^{\#}$ the "abstract predicate". Syntactically, this amounts to replacing any instance of $P($ expr $)$ by $\forall a^{\#}, F_{\sigma}\left(a^{\#}, \operatorname{expr}\right) \rightarrow P^{\#}\left(a^{\#}\right)$.

Algorithm 1 (dataabs $\left(\mathfrak{C}, P, P^{\#}, F_{\sigma}\right)$ )

Input : $\mathfrak{C}:$ Horn clauses; $P$ : predicate to abstract; $P^{\#}:$ unused predicate; $F_{\sigma}$. Computation : for each clause $C \in \mathfrak{C}$, for each $P($ expr $)$ in $C$, replace $P($ expr $)$ by $\forall a^{\#}, F_{\sigma}\left(a^{\#}\right.$, expr $) \rightarrow P^{\#}\left(a^{\#}\right)$, where $a^{\#}$ is a new unused variable.

Example 6 (Using Algorithm 1 to abstract array a of Example 2 with Cell 1 ). Let us define the data abstraction $F_{\sigma_{C e l l} \cdot \sigma_{i d}^{2}}$ (discussed in Section 3.2 by : $F_{\sigma_{\text {Cell }_{1}} \cdot \sigma_{i d}^{2}}\left(\left(i, v, N^{\#}, k^{\#}\right),(a, N, k)\right) \equiv v=a[i] \wedge N^{\#}=N \wedge k^{\#}=k$. And let us execute dataabs(Clauses of Example 2, For $2, \boldsymbol{F o r 2} \mathbf{2}^{\#}, \boldsymbol{F}_{\boldsymbol{\sigma}_{\text {Cell }_{1}} \cdot \boldsymbol{\sigma}_{\text {id }}^{2}}$ ). The result for Clause 4 For $2(a, N, k) \wedge k<N \rightarrow \operatorname{For} 2(a[k \leftarrow a[k]+1], N, k+1)$ is

$$
\begin{gathered}
\left(\forall\left(i^{\#}, v^{\#}, N^{\#}, k^{\#}\right), v^{\#}=a\left[i^{\#}\right] \wedge N^{\#}=N \wedge k^{\#}=k \rightarrow F \text { For } 2^{\#}\left(i^{\#}, v^{\#}, N^{\#}, k^{\#}\right)\right) \wedge k<N \\
\rightarrow\left(\forall\left(i^{\prime \prime}, v^{\prime \#}, N^{\prime \#}, k^{\prime \#}\right), v^{\prime \#}=a[k \leftarrow a[k]+1]\left[i^{\prime \#}\right] \wedge N^{\prime \#}=N \wedge k^{\prime \#}=k+1\right. \\
\left.\rightarrow F o r 2^{\#}\left(i^{\prime \#}, v^{\prime \#}, N^{\prime \#}, k^{\prime \#}\right)\right)
\end{gathered}
$$

where $a^{\#}$ from Algorithm 1 is named $\left(i^{\#}, v^{\#}, N^{\#}, k^{\#}\right)$ in the first replacement and $\left(i^{\prime \#}, v^{\prime \#}, N^{\prime \#}, k^{\prime \#}\right)$ in the second. 
Theorem 5 (Algorithm 1 is correct). If $P^{\#}$ unused in $\mathfrak{C}$, $\left.\forall \mathcal{M}^{\#}, H_{\text {dataabs }(\mathfrak{C}, P, P}, F_{\sigma}\right)\left(\mathcal{M}^{\#}\left[P^{\#} \leftarrow P\right]\right)=\operatorname{abs}\left(\mathcal{G}_{\sigma}^{P}, H_{\mathfrak{C}}\right)\left(\mathcal{M}^{\#}\right)$. Thus, the dataabs algorithm is complete relative to $\mathcal{G}_{\sigma}^{P}$.

When given a set of Horn clauses, one can abstract several predicates (i.e. several program points), perhaps all of them, by applying the abstraction algorithm to them, not necessarily with the same abstraction.

\subsection{Combining data abstractions}

In Example 6, we had to manually adapt the abstraction $\mathrm{Cell}_{1}$ to the predicate For 2 which contained three variables. We define combinators for abstractions such that those adapted abstractions can be easily defined, and later, analyzed.

Definition $9\left(\sigma_{i d}, \sigma_{\perp}, \sigma_{1} \cdot \sigma_{2}, \sigma_{1} \circ \sigma_{2}\right)$. These abstractions and combinators are defined by

1. $\sigma_{i d}(x)=\{x\} ; F_{\sigma_{i d}}\left(x^{\#}, x\right) \equiv x^{\#}=x$.

2. $\sigma_{\perp}(x)=\{\perp\} ; F_{\sigma_{\perp}}\left(x^{\#}, x\right) \equiv x^{\#}=\perp$

3. $\sigma_{1} \cdot \sigma_{2}\left(x_{1}, x_{2}\right)=\sigma_{1}\left(x_{1}\right) \times \sigma_{2}\left(x_{2}\right) ; F_{\sigma_{1} \cdot \sigma_{2}}\left(\left(x_{1}^{\#}, x_{2}^{\#}\right),\left(x_{1}, x_{2}\right)\right) \equiv F_{\sigma_{1}}\left(x_{1}^{\#}, x_{1}\right) \wedge$ $F_{\sigma_{2}}\left(x_{2}^{\#}, x_{2}\right)$

4. $\sigma_{1} \circ \sigma_{2}(x)=\bigcup_{x_{2}^{\#} \in \sigma_{2}\left(x_{2}\right)} \sigma_{1}\left(x_{2}^{\#}\right) ; F_{\sigma_{1} \circ \sigma_{2}}\left(x^{\#}, x\right) \equiv \exists x_{2}^{\#}: F_{\sigma_{1}}\left(x^{\#}, x_{2}^{\#}\right) \wedge F_{\sigma_{2}}\left(x_{2}^{\#}, x\right)$ where $\sigma_{i d}$ is the "no abstraction" abstraction, $\sigma_{\perp}$ abstracts into the unit type (singleton $\perp$ ) and is used with the $\cdot$ combinator to project a variable out, $\sigma_{1} \cdot \sigma_{2}$ abstracts pairs by the cartesian product of their abstractions, and $\sigma_{1} \circ \sigma_{2}$ emulates applying $\sigma_{1}$ after $\sigma_{2}$.

We have given in this section a general scheme for abstracting Horn clauses using data abstraction and shown its correctness. This scheme transforms Horn clauses into extended Horn clauses: new quantifiers $\left(\forall a^{\#}\right)$ are introduced which makes current solvers 11, 17] struggle. We shall now see how to get rid of these quantifiers while retaining relative completeness.

\section{Data Abstraction: Quantifier Removal}

\subsection{A Quantifier Elimination Technique Parametrized by insts}

Contrarily to other approches that use general-purpose heuristics [2], we design our quantifier elimination from the abstraction itself, which allows us to analyze the completeness property of the quantifier elimination algorithm.

The quantifiers introduced by the abstraction scheme are created either by : 1. The $\forall a^{\#}$ of Algorithm 1, which is handled in this paper.

2. Quantifiers within $F_{\sigma}$ (i.e. when abstraction composition is used). In this paper, we only handle existential quantifiers in prenex position of $F_{\sigma}$ which is sufficient for the abstractions of this paper 8 .

${ }^{8}$ In practice this can be expanded by analyzing what the quantifiers within $F_{\sigma}$ would give when moved to prenex position. 
Quantifiers are generated for each instance of the abstracted predicate, and to remove the quantifiers, we separate these instances into two classes :

1. The case when the predicate instance is positive. This case is handled by replacing the quantified variables by free variables, possibly renaming them to avoid name clashes. This is correct as these quantifiers would be universal quantifiers when moved to prenex position and thus have same semantics as free variables when considering the satisfiability of the clauses.

2. The case when the predicate instance is negative. In this case, when moved to prenex position, the quantifiers would be existential and thus can not be easily simplified. We use a technique called quantifier instantiation [2], which replaces a quantifier by that quantifier restricted to some finite set of expressions $I$ called instantiation set (i.e. $\forall a^{\#}$, expr is replaced by $\forall a^{\#} \in I$, expr), which, as $I$ is finite, can be unrolled to remove that quantifier.

Therefore, our quantifier elimination algorithm takes a parameter insts which returns the instantiation set for each abstracted negative predicate instance; and eliminates quantifiers according to their types (negative or positive).

Definition 10 (Instantiation set heuristic insts $\left.\left(F_{\sigma}, a, c t x\right)\right)$. insts is said to be an instantiation set heuristic if and only if:

1. insts takes three parameters : $F_{\sigma}$, the abstraction; a, the variable that was abstracted ; ctx, the context in which the quantifiers are removed.

2. insts $\left(F_{\sigma}, a, c t x\right)$ returns an instantiation set for the pair of quantifiers $a^{\#}$ (the quantified variable corresponding to the abstraction of a) and $q$ (the prenex existential quantifiers of $F_{\sigma}$ ).

Thus its type is a set of pairs of expressions where the first expression is for $a^{\#}$ and the second for the prenex existential quantifiers of $F_{\sigma}$.

To ease the writing of the algorithm, we assume that the input clauses to the quantifier elimination algorithm have been created using the abstraction scheme at most once for each predicate (one may use abstraction composition to emulate applying it twice) on Horn clauses that where initially normalized. Furthermore, as we will be manipulating existential quantifiers within $F_{\sigma}$, we will define $F_{\sigma}[q]$ such that $\left.F_{\sigma}\left(a^{\#}, a\right)=\exists q, F_{\sigma}[q] a^{\#}, a\right)$. In order words, $F_{\sigma}[q]$ is $F_{\sigma}$ where the prenex existential quantifiers have been replaced by the value $q$. We will use () as value for $q$ when $F_{\sigma}$ has no prenex existential quantifiers.

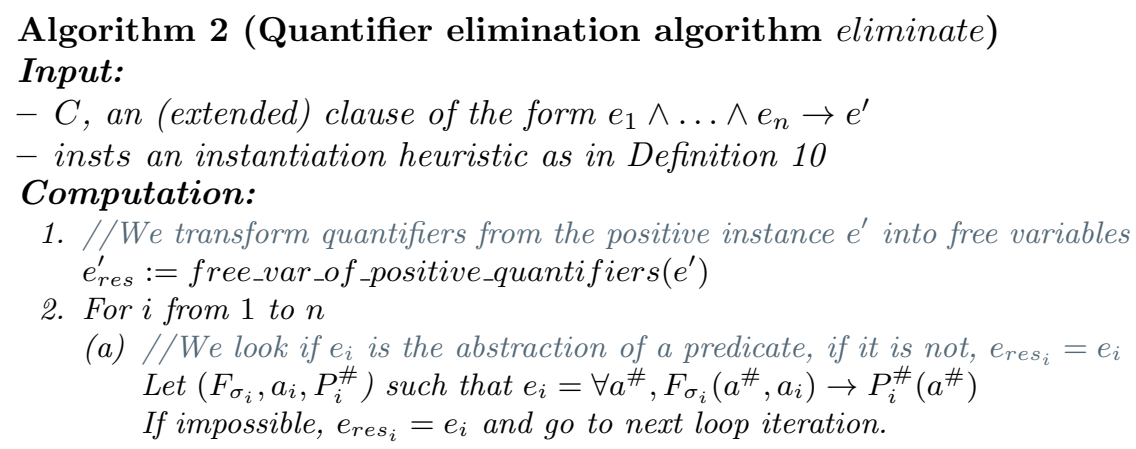


(b) $/ / W e$ compute the context for that instance Let ct $_{i}=e_{\text {res } 1} \wedge \ldots \wedge e_{\text {res }_{i-1}} \wedge e_{i+1} \wedge \ldots \wedge e_{n} \rightarrow e_{\text {res }}^{\prime}$

(c) $/ / W$ compute the instantiation set for that abstraction. Let $I_{i}=\operatorname{insts}\left(F_{\sigma_{i}}, a_{i}\right.$, ctx $\left.x_{i}\right)$

(d) $/ /$ We finally compute $e_{i}$ after instantiation Let $e_{\text {res }_{i}}=\bigwedge_{(a \#, q) \in I_{i}} F_{\sigma_{i}}[q]\left(a^{\#}, a_{i}\right) \rightarrow P_{i}^{\#}\left(a^{\#}\right)$

3. Return $e_{r e s_{1}} \wedge \ldots \wedge e_{r e s_{n}} \rightarrow e_{r e s}^{\prime}$

Example 7 (Eliminating quantifiers of Clause 8 of Example 6). Let us apply eliminate on:

$$
\begin{array}{r}
\forall i^{\#}, v^{\#}, N^{\#}, k^{\#}, v^{\#}=a\left[i^{\#}\right] \wedge N^{\#}=N \wedge k^{\#}=k \rightarrow \operatorname{For} 2^{\#}\left(i^{\#}, v^{\#}, N^{\#}, k^{\#}\right) \wedge k<N \\
\rightarrow \forall i^{\prime \#}, v^{\prime \#}, N^{\prime \#}, k^{\prime \#}, v^{\prime \#}=a[k \leftarrow a[k]+1]\left[i^{\prime \#}\right] \wedge N^{\prime \#}=N \wedge k^{\prime \#}=k+1 \\
\rightarrow F o r 2^{\#}\left(i^{\prime \#}, v^{\prime \#}, N^{\prime \#}, k^{\prime \#}\right)
\end{array}
$$

In this extended clause, $n=2$ an can be decomposed into $e_{1}, e_{2}$ and $e^{\prime}$.

The instantiation algorithm then follows the following steps:

1. Step 1, computes $e_{\text {res }}^{\prime}$ as given in Clause 9

2. We enter Step 2 with $i=1$ and it matches the pattern.

3. We compute the context and call insts (call of Equation 11). Let us assume it returns $\left\{(k, a[k], N, k),\left(i^{\prime \#}, a\left[i^{\prime \#}\right], N, k\right)\right\}$ (which is the value returned by the instantiation set heuristic we construct later in this paper).

4. We get $e_{r e s_{1}}$ as given in Clause 9

5. We enter Step 2 with $i=2$ and it does not match the pattern. Thus, $e_{r_{e s}}=e_{2}$

The final clause is thus

$$
\begin{aligned}
(a[k]= & a[k] \wedge N=N \wedge k=k \rightarrow \operatorname{For}^{\#}(k, a[k], N, k) \wedge \\
& \left.a\left[i^{\prime \#}\right]=a\left[i^{\prime \#}\right] \wedge N=N \wedge k=k \rightarrow F_{\text {or }} 2^{\#}\left(i^{\prime \#}, a\left[i^{\prime \#}\right], N, k\right)\right) \wedge k<N \\
\rightarrow & v^{\prime \#}=a[k \leftarrow a[k]+1]\left[i^{\prime \#}\right] \wedge N^{\prime \#}=N \wedge k^{\prime \#}=k+1 \rightarrow \operatorname{For}^{\#}\left(i^{\prime \#}, v^{\prime \#}, N^{\prime \#}, k^{\prime \#}\right)
\end{aligned}
$$

where $v^{\prime \#}, i^{\prime \#}, N^{\prime \#}, k^{\prime \#}$ are new free variables of the clause.

\section{Simplifying this clause for readability yields:}

$$
\begin{aligned}
\operatorname{For} 2^{\#}(k, a[k], N, k) \wedge F o r 2^{\#} & \left(i^{\prime \#}, a\left[i^{\prime \#}\right], N, k\right) \wedge k<N \\
& \rightarrow F o r 2^{\#}\left(i^{\prime \#}, a[k \leftarrow a[k]+1]\left[i^{\prime \#}\right], N, k+1\right)
\end{aligned}
$$

The call to insts, which will be studied in Examples 8 and 10, was:

$$
\begin{aligned}
\operatorname{insts}\left(F_{\sigma_{C e l l_{1}} \cdot \sigma_{i d}^{2}},(a,(N, k)), e_{2} \rightarrow e_{\text {res }}^{\prime}\right) \\
=\operatorname{insts}\left(F_{\sigma_{C e l l_{1}} \cdot \sigma_{i d}^{2}},(a,(N, k)), k<N \rightarrow v^{\prime \#}=a[k \leftarrow a[k]+1]\left[i^{\prime \#}\right]\right. \\
\left.\wedge N^{\prime \#}=N \wedge k^{\prime \#}=k+1 \rightarrow \operatorname{For}^{\#}\left(\left(i^{\prime \#}, v^{\prime \#}\right),\left(N^{\prime \#}, k^{\prime \#}\right)\right)\right)
\end{aligned}
$$

Theorem 6 (eliminate sound). $\forall C$, insts, $\mathcal{M}, \llbracket$ eliminate $(C$, insts $) \rrbracket_{\mathcal{M}} \Rightarrow \llbracket C \rrbracket_{\mathcal{M}}$ 


\subsection{Constructing a good heuristic insts}

To ensure relative completeness, and thus the predictability, of our overall abstraction (multiple calls to dataabs with different predicates followed by a call to eliminate), we need eliminate to be complete. The completeness of eliminate is highly tied to each call to insts, and we therefore define completeness of a call to insts such that whenever all calls are complete, eliminate is complete.

Definition 11 (Completeness of a call to insts).

We say that a call insts $\left(F_{\sigma}, a\right.$, ctx $)$ is complete if and only if, for any $\mathcal{M}$, and any set $E$ of elements of the types of a, 12 implies 13.

$$
\begin{array}{r}
\forall \text { vars },\left(\forall\left(a^{\#}, q\right), F_{\sigma}[q]\left(a^{\#}, a(\text { vars })\right) \Rightarrow a^{\#} \in \alpha_{\sigma}(E)\right) \Rightarrow \llbracket c t x(\text { vars }) \rrbracket_{\mathcal{M}} \\
\forall \text { vars, }\left(\forall\left(\left(a^{\#}, q\right)\right) \in \operatorname{insts}\left(F_{\sigma}, a, \text { ct }\right)(\text { vars }),\right. \\
\left.F_{\sigma}[q]\left(a^{\#}, a(\text { vars })\right) \Rightarrow a^{\#} \in \alpha_{\sigma}(E)\right) \Rightarrow \llbracket c t x(\text { vars }) \rrbracket_{\mathcal{M}}
\end{array}
$$

Remark 3. We always have 13 implies 12; soundness is based on this.

Remark 4. 12 should be understood as the clause before $\left(a^{\#}, q\right)$ is instantiated: $\alpha_{\sigma}(E)$ represents $\alpha(\mathcal{M})(P)$. Therefore, 12 is similar to $e_{i} \rightarrow$ ctx (of algorithm eliminate) which is the currified current state of the clause in the loop. 13 should be understood as $e_{\text {res }} \rightarrow$ ctx

Theorem 7 (Completeness of insts implies that of eliminate).

For any $C$, insts, $\mathcal{M}$, if during execution of eliminate $(C$, insts) all calls to insts are complete, then $\llbracket C \rrbracket_{\alpha_{\mathcal{G}}(\mathcal{M})}=\llbracket$ eliminate $(C$, insts $) \rrbracket_{\alpha_{\mathcal{G}}(\mathcal{M})}$ where $\mathcal{G}$ is such that $\forall i, \gamma_{\mathcal{G}}\left(\mathcal{M}\left(P_{i}^{\#}\right)\right)=\gamma_{\sigma_{i}}\left(P_{i}^{\#}\right)$, with $i, P_{i}^{\#}, \sigma_{i}$ as defined in eliminate.

Remark 5. We only consider abstract models, that is, $\alpha_{\mathcal{G}}(\mathcal{M})$ where $\mathcal{G}$ represents the galois connection after multiple calls to dataabs. The result is then a consequence of Remark 4

Although our previous completeness definition of insts correctly captures the necessary properties for our instantiation algorithm to keep equisatisfiability, it is too weak to reason on when using combinators (see Section 3.2). The desired property of the instantiation heuristic is what we call strong completeness.

Strong Completeness The definition of completeness only applies in the context of boolean types, as required by the Algorithm eliminate. However, when handling the impact of the instantiation of a quantifier, one wishes to handle that impact with respect to an arbitrarily typed expression. For example, in the case of combinator $\sigma_{1} \cdot \sigma_{2}$, the instantiation of the quantifiers generated by the abstraction $\sigma_{1}$ must be aware of its impact on the variables to be abstracted by $\sigma_{2}$. This leads to a definition of strong completeness that allows any expression type as context parameter of insts, and replaces the satisfiability requirement of the context by an equality requirement. 
Definition 12 (Strong completeness of insts $\left(F_{\sigma}, a, c t x\right)$ ). insts $\left(F_{\sigma}, a\right.$, ctx $)$ is said strongly complete if and only if, for any $E$, vars, $\mathcal{M}$,

$$
\begin{array}{r}
\forall\left(\left(a^{\#}, q\right)\right) \in \operatorname{insts}\left(F_{\sigma}, a, c t x\right)(\text { vars }), F_{\sigma}[q]\left(a^{\#}, a(\text { vars })\right) \Rightarrow a^{\#} \in \alpha_{\sigma}(E) \\
\Rightarrow \exists \operatorname{vars}^{\prime},\left(\forall\left(a^{\#}, q\right), F_{\sigma}[q]\left(a^{\#}, a\left(\text { vars }^{\prime}\right)\right) \Rightarrow a^{\#} \in \alpha_{\sigma}(E)\right) \\
\wedge \llbracket \operatorname{ctx}(\text { vars }) \rrbracket_{\mathcal{M}}=\llbracket \operatorname{ctx}\left(\text { vars }^{\prime}\right) \rrbracket_{\mathcal{M}}
\end{array}
$$

Remark 6. This definition is constructed by contraposing that of completeness.

\section{Theorem 8 (Strong completeness implies completeness).}

If ctx is of boolean type,

insts $\left(F_{\sigma}, a\right.$, ctx $)$ strongly complete $\Rightarrow$ insts $\left(F_{\sigma}, a\right.$, ctx $)$ complete

We give now some results that enable to modularly design instantiation heuristics while remaining (strongly) complete.

Algorithm 3 (insts for finite abstractions) When $\sigma(a)$ finite and $F_{\sigma}$ has no existential quantifiers insts $\left(F_{\sigma}, a\right.$, ct $\left.x\right)=\left\{\left(a^{\#},()\right), a^{\#} \in \sigma(a)\right\}$

Thus insts $\left(F_{\sigma_{i d}}, a, c t x\right)=\{(a,())\}$ and insts $\left(F_{\sigma_{\perp}}, a, c t x\right)=\{(\perp,())\}$

Algorithm 4 (insts for combinators) We will use @ for tuple concatenation.

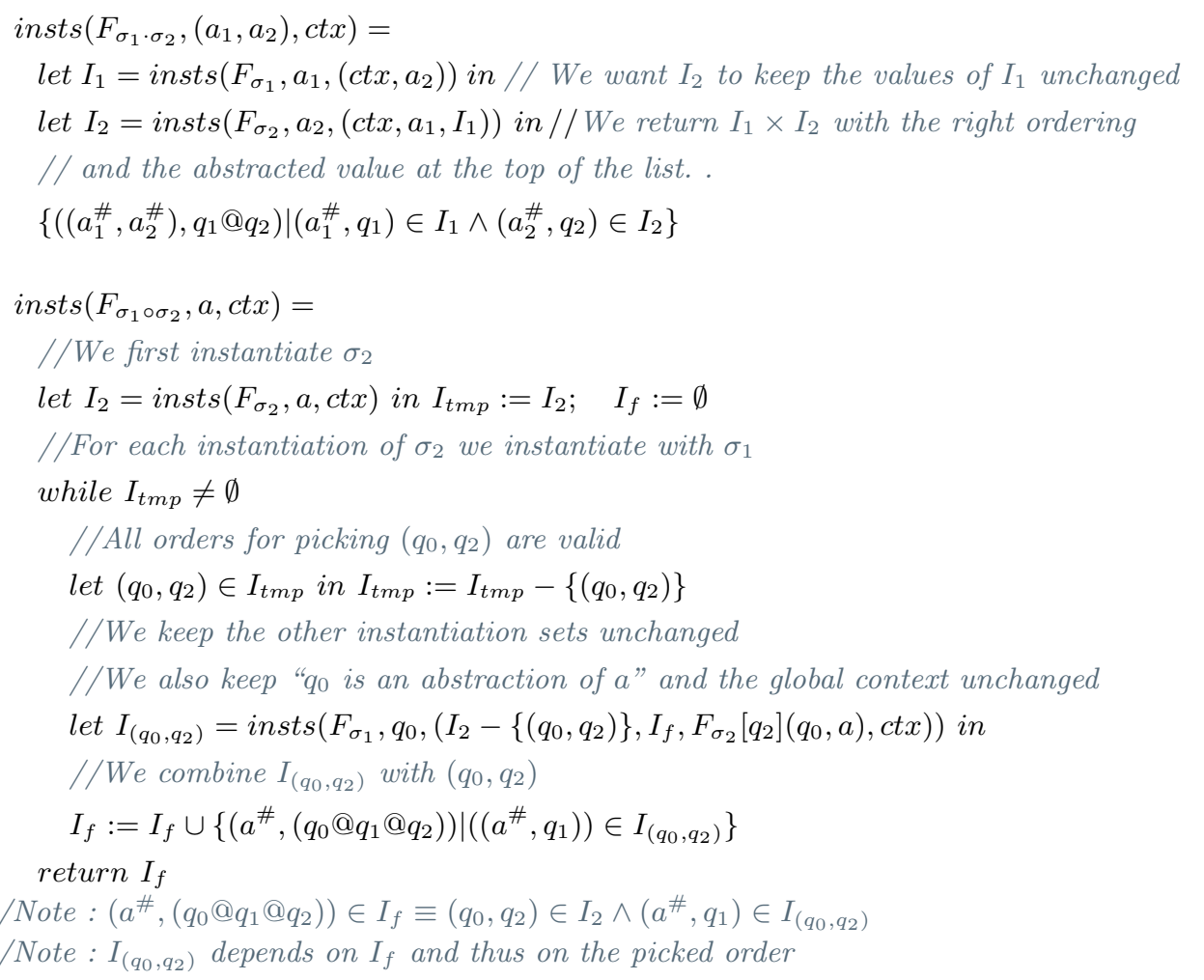


Theorem 9 (Strong Completeness of insts of Algorithms 3 and 4). If $\sigma$ is finite, insts $\left(F_{\sigma}, a, c t x\right)$ is strongly complete. If its recursive calls are strongly complete, insts $\left(F_{\sigma_{1} \cdot \sigma_{2}},\left(a_{1}, a_{2}\right)\right.$, ctx $)$ is strongly complete. If $\sigma_{1}, \sigma_{2}$ are compatible: $\forall E \neq \emptyset, \alpha_{\sigma_{2}} \circ \gamma_{\sigma_{2}} \circ \gamma_{\sigma_{1}} \circ \alpha_{\sigma_{1}} \circ \alpha_{\sigma_{2}}(E)=\gamma_{\sigma_{1}} \circ \alpha_{\sigma_{1}} \circ \alpha_{\sigma_{2}}(E)$ and its recursive calls are strongly complete, then insts $\left(F_{\sigma_{1} \circ \sigma_{2}}, a\right.$, ctx $)$ is strongly complete.

Remark \%. The compatibility condition is true for our abstractions (Theorem 10).

Example 8 (Using combinator instantiation).

In Example 7, we assumed the result of Call 11 .

$$
\begin{aligned}
\operatorname{insts}\left(F_{\sigma_{C e l l_{1}} \cdot \sigma_{i d}^{2}},(a,(N, k)), k<N \rightarrow v^{\prime \#}=a[k \leftarrow a[k]+1]\left[i^{\prime \#}\right]\right. \\
\left.\wedge N^{\prime \#}=N \wedge k^{\prime \#}=k+1 \rightarrow F o r 2^{\#}\left(\left(i^{\prime \#}, v^{\prime \#}\right),\left(N^{\prime \#}, k^{\prime \#}\right)\right)\right)
\end{aligned}
$$

Let us now expand this call further using our combinator construction for insts.

1. We enter the call insts $\left(F_{\sigma_{1} \cdot \sigma_{2}},\left(a_{1}, a_{2}\right)\right.$, ctx $)$ with $\sigma_{1}=\sigma_{\text {Cell }_{1}}, \sigma_{2}=\sigma_{i d}^{2}, a_{1}=$ $a, a_{2}=(N, k)$ and $c t x=k<N \rightarrow v^{\prime \#}=a[k \leftarrow a[k]+1]\left[i^{\prime \#}\right] \wedge N^{\prime \#}=N \wedge k^{\prime \#}=$ $k+1 \rightarrow$ For $^{\#}\left(\left(i^{\prime \#}, v^{\prime \#}\right),\left(N^{\prime \#}, k^{\prime \#}\right)\right)$

2. We compute $I_{1}=\operatorname{insts}\left(F_{\sigma_{1}}, a_{1},\left(c t x, a_{2}\right)\right)$ As we do not have yet an instantiation heuristic for $\sigma_{\mathrm{Cell}_{1}}$, let us assume this call returns $I_{1}=\left\{(k, a[k]),\left(i^{\prime \#}, a\left[i^{\prime \#}\right]\right)\right\}$. This call is further expanded in Example 10.

3. We now compute $I_{2}=\operatorname{insts}\left(F_{\sigma_{2}}, a_{2},\left(\operatorname{ct} x, a_{1}, I_{1}\right)\right)$ But $\sigma_{2}=\sigma_{i d}^{2}$ thus yielding an embedded call to the $\cdot$ combinator

(a) We enter the call insts $\left(F_{\sigma_{i d} \cdot \sigma_{i d}},(N, k),\left(c t x, a_{1}, I_{1}\right)\right)$

(b) We call insts $\left(F_{\sigma_{i d}}, N,\left(\left(c t x, a_{1}, I_{1}\right), k\right)\right)$, yielding $\{N\}$

(c) We call insts $\left(F_{\sigma_{i d}}, k,\left(\left(\right.\right.\right.$ ctx $\left.\left.\left., a_{1}, I_{1}\right), N,\{N\}\right)\right)$, yielding $\{k\}$

(d) We return $\{N, k\}$

4. We return the final result: $\left\{((k, a[k]),(N, k)),\left(\left(i^{\prime \#}, a\left[i^{\prime \#}\right]\right),(N, k+1)\right)\right\}$

Note that if the call to the instantiation using $\mathrm{Cell}_{1}$ is strongly complete, then our final instantiation set is as well. The following call to the instantiation of $\mathrm{Cell}_{1}$ is studied in Example 10.

$$
\begin{aligned}
& \operatorname{insts}\left(F_{\sigma_{1}}, a,\left(\operatorname{ctx}, a_{2}\right)\right)=\left(\operatorname { i n s t s } \left(F_{\sigma_{\text {Cell }_{1}}}, a,\left(k<N \rightarrow v^{\prime \#}=a[k \leftarrow a[k]+1]\left[i^{\prime \#}\right]\right.\right.\right. \\
& \left.\left.\left.\wedge N^{\prime \#}=N \wedge k^{\prime \#}=k+1 \rightarrow \operatorname{For}^{\#}\left(\left(i^{\prime \#}, v^{\prime \#}\right),\left(N^{\prime \#}, k^{\prime \#}\right)\right),(N, k)\right)\right)\right)
\end{aligned}
$$

\section{Cell Abstraction: a complete Data Abstraction}

To illustrate our data abstraction technique, we show how to handle the cell abstractions of Monniaux and Gonnord [16].

\subsection{Cell Abstractions}

Cell abstractions consist in viewing arrays (maps from an index type to a value type) by a finite number of their cells. However, instead of using cells at specific fixed indices, such as the first or the last, we use parametric cells (i.e. cells with a non fixed index). $\mathrm{Cell}_{1}$ of Example 5 corresponds to one parametric cell. In Definition 13 , we extend $C e l l_{1}$ to $\mathrm{Cell}_{n}$. 
Table 1: Properties specified by cell abstractions

\begin{tabular}{|c|c|c|}
\hline Concrete & Abs & Abstract property \\
\hline$a[0]=0$ & Cell $_{1}$ & $i_{1}=0 \Rightarrow v_{1}=0$ \\
\hline$a[n]=0$ & Cell $_{1}$ & $i_{1}=n \Rightarrow v_{1}=0$ \\
\hline$a[0]=a[n]$ & Cell $_{2}$ & $\left(i_{1}=0 \wedge i_{2}=n\right) \Rightarrow v_{1}=v_{2}$ \\
\hline$\forall i, a[i]=0$ & Cell $_{1}$ & $v_{1}=0$ \\
\hline$\forall i, a[i]=i^{2}$ & Cell $_{1}$ & $v_{1}=i_{1}^{2}$ \\
\hline$\forall i, a[n] \geq a[i]$ & Cell $_{2}$ & $i_{2}=n \Rightarrow v_{2} \geq v_{1}$ \\
\hline
\end{tabular}

Definition 13. Cell abstractions Cell $_{n}$.

$\left.\sigma_{\text {Cell }_{n}}(a)=\left\{\left(i_{1}, a\left[i_{1}\right]\right), \ldots,\left(i_{n}, a\left[i_{n}\right]\right)\right)\right\}$ and $F_{\sigma_{C e l l_{n}}}\left(\left(\left(i_{1}, v_{1}\right), \ldots,\left(i_{n}, v_{n}\right)\right), a\right) \equiv v_{1}=a\left[i_{1}\right] \wedge \ldots \wedge v_{n}=a\left[i_{n}\right]$.

Many interesting properties can be defined by cell abstractions (Tab. 1). Furthermore, our data abstraction framework allows formalizing other existing array abstractions by compositions of cell abstractions (Example 9).

Example 9. Array abstractions from cell abstractions.

Array smashing: $\sigma_{\text {smash }}(a)=\{a[i]\}$. This abstraction keeps the set of values reached but loses all information linking indices and values. It can be constructed using $C e l l_{1}$ abstraction in the following way : $\left.\sigma_{\text {smash }} \equiv\left(\sigma_{\perp} \cdot \sigma_{i d}\right) \circ \sigma_{C e l l}\right]^{9}$

Array slicing: 5.710 There are several variations, and for readability we present the one that corresponds to "smashing each slice" and picking the slices ] $\infty, i[,[i, i]] i,, \infty\left[: \sigma_{\text {slice }}(a)=\left\{\left(a\left[j_{1}\right], a[i], a\left[j_{3}\right]\right), j_{1}<i \wedge j_{3}>i\right\}\right.$. It can be constructed using Cell $_{1}$ abstraction in the following way : $\sigma_{\text {slice }} \equiv\left(\sigma_{\text {slice }_{1}} \cdot \sigma_{\text {slice }_{2}}\right.$. $\left.\sigma_{\text {slice }_{3}}\right) \circ \sigma_{\text {Cell }_{3}}{ }^{10}$ with $\sigma_{s_{k}}(j, v)=i t e\left(j \in\right.$ slice $\left._{k}, v, \perp\right)$

Theorem 10. The abstractions of Example 9 have strongly complete instantiation heuristics when $\mathrm{Cell}_{n}$ has.

Remark 8. These abstractions are of the form $\sigma \circ \sigma_{\text {Cell }_{n}}$. Their strong completeness is proven because $\sigma$ is finite and $\sigma_{C e l l_{n}}$ always verifies the compatibility condition of Theorem 9 when left-side composed.

\subsection{Instantiating Cell Abstractions}

The data abstraction framework requires an instantiation heuristic insts for $\mathrm{Cell}_{n}$. To achieve the strong completeness property, we first compute all "relevant" indices of the abstracted array, that is, indices which when left unchanged ensure that ctx keeps its value.

\footnotetext{
${ }^{9}$ This is a semantic equivalence, not a strict equality : formally $\left(\sigma_{\perp} \cdot \sigma_{i d}\right) \circ \sigma_{C e l l_{1}}(a)$ returns $\{(\perp, a[i]\}$ instead of $\{a[i]\}$

${ }^{10}$ As for smashing, this is a semantic equivalence
} 


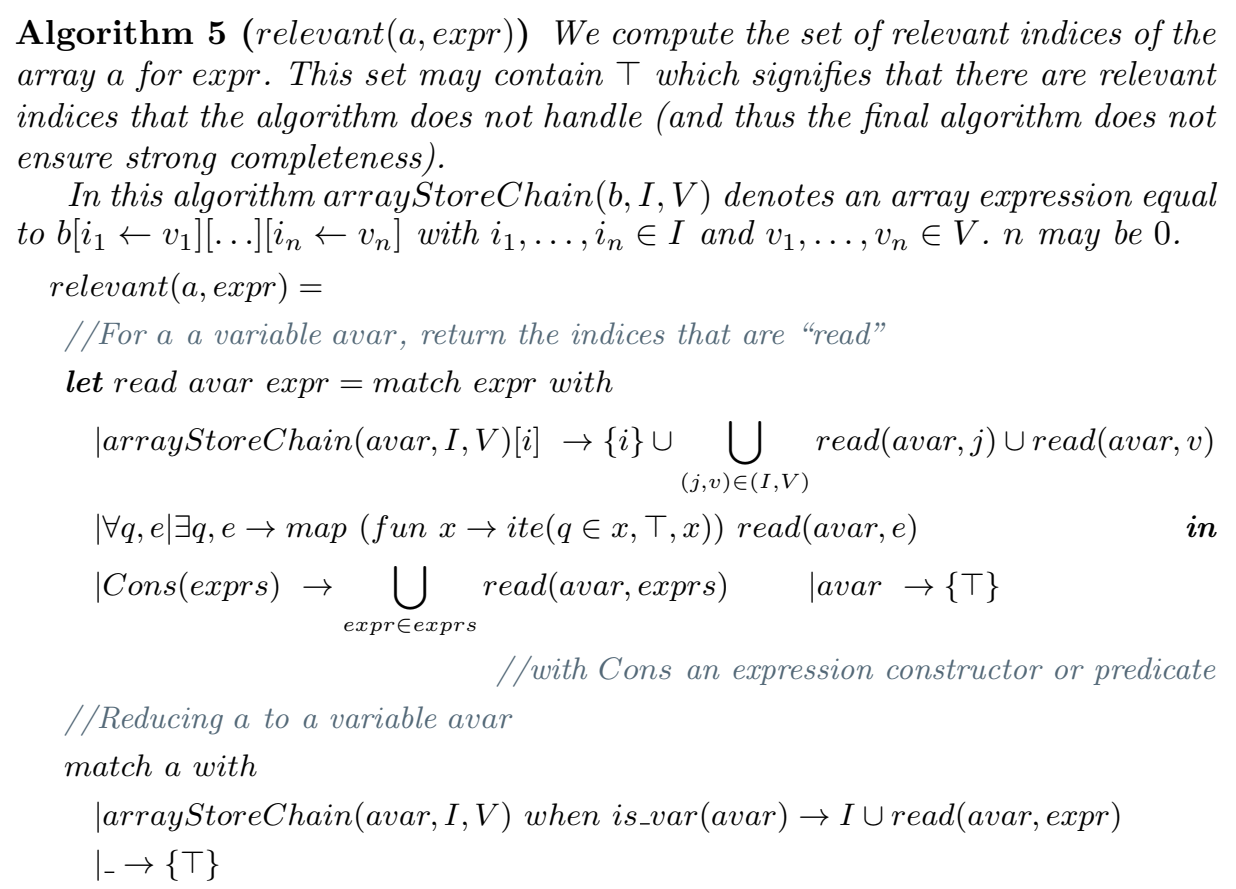

Remark 9. For readability, the algorithm is kept short, but may be improved to return $T$ in fewer cases using techniques from $[3$

Theorem 11 (relevant (a, expr) is correct). If $\top \notin$ relevant $(a$, expr $)$ then $\forall \mathcal{M}$, vars, $a^{\prime},\left(\forall i \in\right.$ relevant $(a$, expr $), a^{\prime}[i]=a($ vars $\left.)[i]\right) \Rightarrow$

$$
\exists \operatorname{var} s^{\prime}, \llbracket \operatorname{expr}(\operatorname{vars}) \rrbracket_{\mathcal{M}}=\llbracket \operatorname{expr}\left(\operatorname{vars}^{\prime}\right) \rrbracket_{\mathcal{M}} \wedge a\left(\operatorname{vars}^{\prime}\right)=a^{\prime}
$$

We use our relevant indices to construct an instantiation heuristic for $\mathrm{Cell}_{n}$. Algorithm 6 (Instantiation heuristic for $\mathrm{Cell}_{n}$ )

$$
\begin{aligned}
& \text { insts }\left(F_{\sigma_{C e l l_{n}}}, a, \text { ctx }\right)= \\
& \text { let } R=\text { relevant }(a, \text { ctx }) \text { in } \quad \text { //Compute relevant set } \\
& \text { let Ind }=\text { ite }(R=\emptyset,, R-\{\top\}) \text { in } \quad \text { //Make it non empty and remove } \top \\
& / / \text { can be chosen as any value of the index type } \\
& \text { let } I=\{(i, a[i]) \mid i \in I n d\} \text { in }\left(I^{n},()\right) / / \text { make it a pair index value, make } n \text { copies }
\end{aligned}
$$

Theorem 12 (Strong Completeness for cell Abstraction). Any call to insts $\left(F_{\sigma_{\text {Cell }_{n}}}, a, c t x\right)$ is strongly complete whenever $\top \notin$ relevant $(a$, ct $x)$.

Example 10 (Instantiation of $\mathrm{Cell}_{1}$ ).

In Example 8, we assumed the result of the Call 14

$$
\begin{aligned}
& \operatorname{insts}\left(F_{\sigma_{1}}, a,\left(\operatorname{ctx}, a_{2}\right)\right) \\
& \quad=\operatorname{insts}\left(F_{\sigma_{\text {Cell }_{1}}}, a,\left(k<N \rightarrow v^{\prime \#}=a[k \leftarrow a[k]+1]\left[i^{\prime \#}\right]\right.\right. \\
& \left.\left.\wedge N^{\prime \#}=N \wedge k^{\prime \#}=k+1 \rightarrow \operatorname{For}^{\#}\left(\left(i^{\prime \#}, v^{\prime \#}\right),\left(N^{\prime \#}, k^{\prime \#}\right)\right),(N, k)\right)\right)
\end{aligned}
$$


Let us use our instantiation heuristic for $\mathrm{Cell}_{1}$ to compute the result of that call.

1. We first compute the relevant set, we obtain $R=\left\{k, i^{\prime \#}\right\}$

2. It is already non empty and does not contain $\top$, so Ind $=R=\left\{k, i^{\prime \#}\right\}$

3 . We add the value part, yielding $I=\left\{(k, a[k]),\left(i^{\prime \#}, a\left[i^{\prime \#}\right]\right)\right\}$

4. $n=1$, therefore the result is $I^{1}=I=\left\{(k, a[k]),\left(i^{\prime \#}, a\left[i^{\prime \#}\right]\right)\right\}$

And the call is complete as the relevant set did not contain $T$.

\section{Implementation and Experiments}

In Sections 3 and 4 , we constructed the building blocks to verify programs using an abstraction technique that strives to ensure relative completeness and in Section 5. we gave a powerful abstraction for arrays. We now combine these sections to create a tool for the verification of programs with arrays. We benchmark this tool and compare it to other tools and then analyze the results.

\subsection{The full algorithm}

Our tool uses Algorithm 7 which has for only parameter $n$. The abstraction used consists in abstracting each array of each predicate by $\mathrm{Cell}_{n}$.

\section{Algorithm 7 (Full $n$-Cell Abstraction Algorithm $\operatorname{tr} s(\mathfrak{C}, n)$ )}

Input: $A$ set of Horn clauses $\mathfrak{C}, n$ the number of cells

\section{Computation:}

1. Abstraction: For each predicate $P$ of $C$ over types $t_{1}, \ldots, t_{k}$

(a) Let $^{P}=\prod_{1 \leq i \leq k}$ ite $\left(i \operatorname{Array}\left(t_{k}\right), \sigma_{\text {Cell }_{n}}, \sigma_{i d}\right)$

(b) Let $P^{\#}$ be an unused predicate.

(c) $\mathfrak{C}:=\operatorname{dataabs}\left(\mathfrak{C}, F_{\sigma}^{P}, P, P^{\#}\right)$

2. Quantifier elimination: For each clause $C \in \mathfrak{C}$

$C:=$ eliminate $(C$, insts $) / /$ insts is the heuristic discussed in this article

3. Simplification (optional): For each clause $C \in \mathfrak{C}$

(a) $C:=$ simplify $(C$, insts $) / /$ we simplify as in Clause 10

(b) $C:=$ Ackermannize $(C) /{ }^{*}$ This step removes possible array writes by applying read over write axioms. Then if an array a is only used in read expressions, we eliminate it at quadratic cost [14, \$3.3.1]. Note that this is is not possible before abstraction because a is a parameter of predicates. */

We implemented this algorithm, and used it on clauses generated by the Vaphor converter from mini-Java programs to Horn Clauses. The implementation is publicly available ${ }^{11}$. Overall the toolchain ensures soundness and relative completeness due to the strong completeness of our building blocks. To ensure that within the computation of insts $\left(F_{\sigma_{\text {Cell }_{n}}}, a, c t x\right)$, relevant $(a, c t x)$ does not contain $T$, we rely on the form of clauses generated by the Vaphor converter,

11 https://github.com/vaphor 
Table 2: Experimental results

\begin{tabular}{|c|c|c|c|c|c|c|c|c|c|c|c|c|c|c|c|c|}
\hline & \multirow{2}{*}{\multicolumn{2}{|c|}{$\# \operatorname{prg} r$}} & \multicolumn{4}{|c|}{ Noabs } & \multicolumn{2}{|c|}{ VapHor } & \multicolumn{4}{|c|}{ Dataabs } & \multicolumn{4}{|c|}{ Dataabs acker } \\
\hline & & & 6 & i & $?$ & $\geq 1$ & 6 ? & $\geq 1$ & $b$ & i & $?$ & $\geq 1$ & 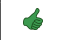 & 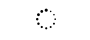 & $?$ & $\geq 1$ \\
\hline Buggy & 4 & 1 & 4 & 0 & 0 & 4 & $\begin{array}{lll}4 & 0 & 0\end{array}$ & 4 & 4 & 0 & 0 & 4 & 4 & 0 & 0 & 4 \\
\hline Buggy & 4 & 2 & - & - & - & - & $4 \begin{array}{lll}4 & 0 & 0\end{array}$ & 4 & 0 & 4 & 0 & 0 & 3 & 1 & 0 & 3 \\
\hline NotHinted & 12 & 1 & 01 & 11.5 & 0.5 & 0 & 1110 & 1 & 0 & 12 & 0 & 12 & 0 & 11.83 & 0.17 & 0 \\
\hline NotHinted & 12 & 2 & - & - & - & - & $\begin{array}{lll}0 & 12 & 0\end{array}$ & 0 & 0 & 12 & 0 & 12 & 0 & 12 & 0 & 0 \\
\hline Hinted & 12 & 1 & 0 & 11 & 1 & 0 & $\begin{array}{lll}4 & 8 & 0\end{array}$ & 7 & 8.99 & 2.84 & 0.17 & 11 & 8.83 & 3.17 & 0 & 12 \\
\hline Hinted & 12 & 2 & - & - & - & - & $\begin{array}{lll}0 & 12 & 0\end{array}$ & 0 & 0 & 12 & 0 & 0 & 5.83 & 6.17 & 0 & 6 \\
\hline
\end{tabular}

Columns: corresponds to the abstraction tool. 1. Noabs corresponds to no abstraction

2. Vaphor is the tool by Monniaux \& Gonnord 3. Dataabs and Dataabs acker represent our implementation, with and without Ackermannisation.

Lines: files grouped by category and number of cells

Values:(respectively, ?) correspond to the number of files that where correct (respectively timeout, unknown), averaged over the 3 naming options and the 2 random seeds). $\geq 1$ represents the number of files for which at least one of the naming options and random seeds returned the correct result.

among which: 1 . There are no array equalities. 2 . There is at most one negative predicate per clause. Theorem 14 available in the appendix states the exact requirements on the form of the clauses to ensure relative completeness.

\subsection{Experimental setting and results}

We modified the converter to take into account optional additional invariant information given by the programmer, in the form of "hints". These hints are additional assertion clauses so that the solver may converge more easily to the desired model. These additional clauses are also abstracted by our abstraction.

Our initial Horn clauses are generated by the converter from programs in three categories: 1 . incorrect programs. 2. correct programs without hints. 3. correct programs with hints. We then compare our implementation of Algorithm 7 with no abstraction and the abstraction tool of Vaphor on the modified benchmarks of 16. Note that the latter tool implements a variant of $\mathrm{Cell}_{n}$ that always Ackermannizes in the process. We modified the benchmarks of [6, 16, such that properties are checked using a loop instead of a random access, e.g., they take $i$ random and assert $t[i]=0$ to verify $\forall i t[i]=0$, thus enforcing the use of quantified invariants. This is why Vaphor behaves much more poorly on our examples than in 16]. The modified benchmarks are available at https://github.com/vaphor/array-benchmarks.

We use as backend the state of the art solver Z3 version 4.8 .8 - 64 bit with timeout 40s on a laptop computer. Because of issue https://github.com/ Z3Prover/z3/issues/909 that we have witnessed, we launch each benchmark with 3 different naming conventions for predicates in addition to using 2 random seeds. The results are presented in Table 2. We did not show columns for incorrect results or errors as there are none. 


\subsection{Analysis}

Abstraction 8 Solving timings Our abstraction is fast: most of it is search \& replace; most of the time is spent in the hand-written simplification algorithm, which is not the purpose of this paper. The solving time in Z3 is much higher. It seems that, for correct examples, Z3 either converges quickly $(<5 \mathrm{sec})$ or fails to converge regardless of timeout - only in 18 cases out of 1176 does it solve the problem between $5 \mathrm{~s}$ and the timeout.

Soundness and relative completeness There are no incorrect results, confirming that all implementations are sound. There are cases where Z3 cannot conclude "correct" on its own but with enough help (i.e. hints and Ackermannization, different predicate names, and random seeds) all files are solved as shown by the hinted, $\mathrm{Cell}_{1}$ line, column $\geq 1$ of Dataabs acker. This confirms that our implementation is relatively complete, as proved in theory.

Tool comparison $\mathrm{Z} 3$ without abstraction is unable to solve any correct example, even with hints. We mostly behave better than Vaphor for Cell 1 on hinted examples, perhaps because we create smaller instantiation sets: Vaphor handles complex clauses greedily, using all indices read as instantiation set.

Cell $_{1}$ versus Cell $_{2}$ All our correct examples have invariants expressible by $\mathrm{Cell}_{1}$, and thus also by $\mathrm{Cell}_{2}$. However, the clauses generated using $\mathrm{Cell}_{2}$ are bigger and the instantiation set sizes are squared, thus complexifying the clauses and probably stopping Z3 from converging easily on an invariant. Vaphor, in contrast, generates fewer instantiations for Cell2 by using $\sigma_{\text {Cell }_{2}}(a)=\{(i, a[i], j, a[j]), i<$ $j\}$, which explains its better performance on $\mathrm{Cell}_{2}$ buggy examples.

Ackermannizing or not Ackermannization completely removes arrays from the clauses, but changes neither the invariants nor the space in which invariants are sought. Z3 is supposed to handle the theory of arrays natively and should thus be more efficient than our eager Ackermannization; yet the latter improves results on non buggy examples.

Overall results and Z3 Our tool transforms a Horn problem with arrays requiring quantified invariants which lie within the $\mathrm{Cell}_{n}$ abstraction into an equivalently satisfiable Horn problem which needs neither quantifiers nor arrays. The non-hinted examples show that this is insufficient to make automatic proofs of programs with arrays practical, mainly because Z3 struggles to handle these array free Horn problems. In addition, Z3 sometimes succeeds or not, or even returns unknown, depending on details in the input (e.g., predicate naming, randon, Ackermannisation). Further work is needed on integer Horn solvers for better performance and reduced brittleness.

Benchmarks and future work The current benchmarks are generated from a toy Java langage and have invariants expressible in $\mathrm{Cell}_{1}$. Future work includes (i) adding new challenging examples which require $\mathrm{Cell}_{2}$ such as sortedness or even more complex invariants such as "the array content is preserved as a multiset" [16; (ii) tackling challenging literature examples [1] in real langages, perhaps using a front end such as SeaHorn [8]. 


\section{Related Work}

Numerous abstractions for arrays have been proposed in the literature, among which array slicing [5, 7, 10. In Example 9 we showed how they are expressible in our framework. Similarly to Monniaux and Alberti [15] we think that disconnecting the array abstraction from other abstractions and from solving enables using back-end solvers better. Like Monniaux and Gonnord [16] we use Horn Clauses to encode our program under verification, but we go a step further by using Horn Clauses as an intermediate representation to chain abstractions. Furthermore, our formalization is cleaner for multiple arrays and proves relative completeness.

Our instantiation method is inspired from previous work on solving quantified formulae [2, 3, 9]. 3 does not consider Horn clauses, that is, expressions with unknown predicates, but only expressions with quantifiers. 2 2 has an approach very similar to ours, but without casting it within the framework of data abstractions; they use trigger-based instantiation. Both instantiation methods of [2,3] lead to bigger instantiation sets than the one we suggest, yet, contrary to us, they do not prove completeness. Finally, the technique used in 9 creates instantiation sets not as pre-processing, but during analysis. Although more general, it is highly likely that the technique suffers from the same unpredictability that Horn solvers have. In our case, we believe that we can tailor the instantiation set to the abstraction and analyze its precision.

Finally, other recent approaches focus on more powerful invariants through proofs by induction 12. However, as stated by their authors, their approach is complementary to ours: theirs is less specialized, and thus has trouble where our approach may easily succeed, but enables other invariants: our data abstraction framework may allow abstracting within their induction proofs.

\section{Conclusion}

We have proposed an approach for the definition, combination and solving of data abstractions for Horn Clauses. The framework also provides sufficient conditions for (relative) completeness, and prove the result for a large class of array abstractions. We propose an implementation and experimental evaluation on classical examples of the literature. Future work include extending the applicability of the framework for other data structures such that trees.

\section{References}

1. Beyer, D.: Automatic Verification of C and Java Programs: SV-COMP 2019. In: TACAS (2019)

2. Bjørner, N., McMillan, K., Rybalchenko, A.: On Solving Universally Quantified Horn Clauses. In: SAS (2013)

3. Bradley, A.R., Manna, Z., Sipma, H.B.: What's decidable about arrays? In: VMCAI (2006) 
4. Cousot, P., Cousot, R.: Abstract Interpretation: A Unified Lattice Model for Static Analysis of Programs by Construction or Approximation of Fixpoints. In: POPL (1977)

5. Cousot, P., Cousot, R., Logozzo, F.: A parametric segmentation functor for fully automatic and scalable array content analysis. SIGPLAN Not. 46(1), 105-118 (Jan 2011)

6. Dillig, I., Dillig, T., Aiken, A.: Fluid updates: Beyond strong vs. weak updates. In: Gordon, A.D. (ed.) Proceedings of the 19th European Symposium on Programming. LNCS, vol. 6012, pp. 246-266. Springer (2010)

7. Gopan, D., Reps, T., Sagiv, M.: A Framework for Numeric Analysis of Array Operations. In: PLDI (2005)

8. Gurfinkel, A., Kahsai, T., Komuravelli, A., Navas, J.: The SeaHorn Verification Framework. In: CAV (2015)

9. Gurfinkel, A., Shoham, S., Vizel, Y.: Quantifiers on Demand. In: ATVA (2018)

10. Halbwachs, N., Péron, M.: Discovering Properties About Arrays in Simple Programs. In: Proceedings of the 29th ACM SIGPLAN Conference on Programming Language Design and Implementation (PLDI'08). Association for Computing Machinery, New York, NY, USA (2008)

11. Hojjat, H., Rümmer, P.: The ELDARICA Horn Solver. In: FMCAD (2018)

12. Ish-Shalom, O., Itzhaky, S., Rinetzky, N., Shoham, S.: Putting the Squeeze on Array Programs: Loop Verification via Inductive Rank Reduction. In: VMCAI (2020)

13. Kahsai, T., Rümmer, P., Schäf, M.: JayHorn: A Java Model Checker: (Competition Contribution) (2019)

14. Kroening, D., Strichman, O.: Decision procedures. Springer (2008)

15. Monniaux, D., Alberti, F.: A simple abstraction of arrays and maps by program translation. In: SAS (2015)

16. Monniaux, D., Gonnord, L.: Cell morphing: From array programs to array-free horn clauses. In: Static Analysis - 23rd International Symposium, SAS 2016, Edinburgh, UK, September 8-10, 2016, Proceedings. pp. 361-382 (2016)

17. de Moura, L.M., Bjørner, N.: Z3: An efficient SMT solver. In: TACAS (2008)

18. Rival, X., Yi, K.: Introduction to Static Analysis An Abstract Interpretation Perspective. MIT Press (2020) 


\section{A Appendix: proofs}

\section{A.1 Horn Clauses and Galois connections}

Definition 14 (Galois connection $\mathcal{G}$, defines $\alpha_{\mathcal{G}}, \gamma_{\mathcal{G}}$ ).

$A$ Galois connection $\mathcal{G}$ between two lattices is defined by

$-\alpha_{\mathcal{G}}: \mathscr{C} \rightarrow \mathcal{A}$ gives the abstraction of a value.

- $\gamma_{\mathcal{G}}: \mathcal{A} \rightarrow \mathscr{C}$ gives the concrete value of an abstract element.

where: (i) $\alpha_{\mathcal{G}}, \gamma_{\mathcal{G}}$ are monotone (ii) $a \leq \alpha_{\mathcal{G}}(c) \equiv \gamma_{\mathcal{G}}(a) \leq c$

Theorem 13 (Further properties of Galois connections).

3. $S \leq \gamma_{\mathcal{G}}\left(\alpha_{\mathcal{G}}(S)\right)$ for soundness.

4. $\forall S^{\#}, \alpha_{\mathcal{G}}\left(\gamma_{\mathcal{G}}\left(S^{\#}\right)\right) \leq S^{\#}$ for minimal precision loss.

5. $\alpha_{\mathcal{G}} \circ \gamma_{\mathcal{G}} \circ \alpha_{\mathcal{G}}=\alpha_{\mathcal{G}}$ and $\gamma_{\mathcal{G}} \circ \alpha_{\mathcal{G}} \circ \gamma_{\mathcal{G}}=\gamma_{\mathcal{G}}$ : abstracting an already abstracted element yields that element.

Proof. These proofs can be found in 18 .

Theorem 1 (Horn problems as a condition on models, defines $H(\mathcal{M})$ ). A Horn problem $H$ is satisfiable if and only if $\exists \mathcal{M}, f_{H}(\mathcal{M}) \leq \mathcal{M} \wedge \mathcal{M} \leq \mathcal{U}_{H}$

Proof. Assume $H(\mathcal{M})$. Because $f_{H}(\mathcal{M}) \leq \mathcal{M}, \mathcal{M}$ is a post fixpoint and therefore lfp $f_{H} \leq \mathcal{M}$. Finally, we have $\operatorname{lfp} f_{H} \leq \mathcal{M} \leq \mathcal{U}_{H}$.

Assume lfp $f_{H} \leq \mathcal{U}_{H}$. We have $H\left(\operatorname{lfp} f_{H}\right)$.

Theorem 2 (Horn clauses are instances of Horn problems, defines $H_{\mathfrak{C}}$ ).

Let $\mathfrak{C}$ be a set of Horn clauses. There exists a Horn problem $H_{\mathfrak{C}}$, such that for any model $\mathcal{M}, H_{\mathfrak{C}}(\mathcal{M})=\bigwedge_{C \in \mathfrak{C}} \llbracket \forall$ vars,$C($ vars $) \rrbracket_{\mathcal{M}}$.

Proof. $\bigvee$ denotes the join (least upper bound) operator of the lattice of models, $\bigwedge$ the meet operator (greatest lower bound). Assertion clauses are clauses with no positive predicates. For a set of Horn clauses $\mathfrak{C}$ denote $\operatorname{assert}(\mathfrak{C})$ the set of assertion clauses of $\mathfrak{C}$.

Let $f_{H_{\mathfrak{C}}}(\mathcal{M})=\bigwedge\left\{\mathcal{M}^{\prime} \mid \forall C \in(\mathfrak{C}-\operatorname{assert}(\mathfrak{C})), \llbracket \forall\right.$ vars,$C($ vars $\left.) \rrbracket_{\text {pos }=\mathcal{M}^{\prime}, \text { neg }=\mathcal{M}}\right\}$ where $\llbracket X \rrbracket_{\text {pos }=\mathcal{M}^{\prime}, \text { ne }=\mathcal{M}}$ interprets the positive predicate of $X$ by $\mathcal{M}^{\prime}$ and the negative predicates by $\mathcal{M}$.

Let $\mathcal{U}_{H_{\mathfrak{C}}}=\bigvee\left\{\mathcal{M} \mid \forall C \in \operatorname{assert}(\mathfrak{C}), \llbracket \forall\right.$ vars,$C($ vars $\left.) \rrbracket_{\mathcal{M}}\right\}$.

1. $f_{H_{\mathfrak{C}}}$ monotonic: consider $\mathcal{M}_{1} \leq \mathcal{M}_{2}$.

$\left\{\mathcal{M}^{\prime} \mid \forall C \in(\mathfrak{C}-\operatorname{assert}(\mathfrak{C})), \llbracket \forall\right.$ vars,$C($ vars $\left.) \rrbracket_{\text {pos }=\mathcal{M}^{\prime}, \text { neg }=\mathcal{M}_{2}}\right\} \subseteq\left\{\mathcal{M}^{\prime} \mid \forall C \in(\mathfrak{C}-\right.$ $\operatorname{assert}(\mathfrak{C})), \llbracket \forall$ vars,$C($ vars $\left.) \rrbracket_{\text {pos }=\mathcal{M}^{\prime}, \text { neg }=\mathcal{M}_{1}}\right\}$. Thus, $f_{H_{\mathfrak{C}}}\left(\mathcal{M}_{1}\right) \leq f_{H_{\mathfrak{C}}}\left(\mathcal{M}_{2}\right)$.

2. $H_{\mathfrak{C}}(\mathcal{M})=\forall C \in \mathfrak{C}, \llbracket \forall$ vars,$C($ vars $) \rrbracket_{\mathcal{M}}$ :

(a) Assume $H_{\mathfrak{C}}(\mathcal{M})$.

We have $\forall C \in(\mathfrak{C}-\operatorname{assert}(\mathfrak{C})), \llbracket \forall$ vars,$C(\operatorname{var} s) \rrbracket_{p o s=f_{H_{\mathfrak{C}}}(\mathcal{M}), \text { neg }=\mathcal{M}}$. Thus, because $f_{H_{\mathfrak{C}}}(\mathcal{M}) \leq \mathcal{M}$, we have

$\forall C \in(\mathfrak{C}-\operatorname{assert}(\mathfrak{C})), \llbracket \forall$ vars,$C($ vars $) \rrbracket_{\text {pos }=\mathcal{M}, \text { neg }=\mathcal{M}}$

$=\forall C \in(\mathfrak{C}-\operatorname{assert}(\mathfrak{C})), \llbracket \forall$ vars,$C($ vars $) \rrbracket_{\mathcal{M}}$. The assertion clauses are simply proven by $\mathcal{M} \leq \mathcal{U}_{H_{\mathfrak{e}}}$ 
(b) Assume $\forall C \in \mathfrak{C}, \llbracket \forall$ vars,$C($ vars $) \rrbracket_{\mathcal{M}}$. First $\mathcal{M} \leq \mathcal{U}_{H_{\mathfrak{C}}}$ by definition of $\mathcal{U}_{H_{\mathfrak{C}}}$. Secondly, $\mathcal{M} \in\left\{\mathcal{M}^{\prime} \mid \forall C \in(\mathfrak{C}\right.$-assert $(\mathfrak{C}))$, $\llbracket \forall$ vars,$C($ vars $\left.) \rrbracket_{\text {pos }=\mathcal{M}^{\prime}, \text { neg }=\mathcal{M}}\right\}$, thus $f_{H_{\mathfrak{C}}}(\mathcal{M}) \leq \mathcal{M}$.

Theorem 3 (Definition abs $(\mathcal{G}, H)$ is correct). For all $\mathcal{M}^{\#}$, the following statements are equivalent (with the notation $H(\mathcal{M})$, where $H$ is a Horn problem and $\mathcal{M}$ a possible model, from Theorem 1):

1. $\operatorname{abs}(\mathcal{G}, H)\left(\mathcal{M}^{\#}\right)$

2. $H\left(\gamma_{\mathcal{G}}\left(\mathcal{M}^{\#}\right)\right)$

3. $\operatorname{abs}(\mathcal{G}, H)\left(\alpha_{\mathcal{G}} \circ \gamma_{\mathcal{G}}\left(\mathcal{M}^{\#}\right)\right)$

Remark 10. From this theorem, it follows that:

1. The definition of abs $(\mathcal{G}, H)$ corresponds to the desired abstraction as : $H$ satisfiable by a model expressible by the abstraction $\left(\gamma_{\mathcal{G}}\left(\mathcal{M}^{\#}\right)\right)$ is equivalent to $\operatorname{abs}(\mathcal{G}, H)$ satisfiable.

2. abs $(\mathcal{G}, H)\left(\mathcal{M}^{\#}\right)$ is constructible from $H$ and $\gamma_{\mathcal{G}}$. This will be used in Theorem 5.

Proof. Introduce $\mathcal{M}^{\#}$. Proof that i is equivalent to ii

$$
\begin{aligned}
& \operatorname{abs}(\mathcal{G}, H)\left(\mathcal{M}^{\#}\right) \\
\equiv & \alpha_{\mathcal{G}} \circ f_{H} \circ \gamma_{\mathcal{G}}\left(\mathcal{M}^{\#}\right) \leq \mathcal{M}^{\#} \wedge \mathcal{M}^{\#} \leq \alpha_{\mathcal{G}}\left(\mathcal{U}_{H}\right)
\end{aligned}
$$

We use the item ii of Definition 14

$$
\begin{aligned}
& \equiv f_{H} \circ \gamma_{\mathcal{G}}\left(\mathcal{M}^{\#}\right) \leq \gamma_{\mathcal{G}}\left(\mathcal{M}^{\#}\right) \wedge \gamma_{\mathcal{G}}\left(\mathcal{M}^{\#}\right) \leq \mathcal{U}_{H} \\
& \equiv H\left(\gamma_{\mathcal{G}}\left(\mathcal{M}^{\#}\right)\right)
\end{aligned}
$$

Proof that i is equivalent to iii

$$
\begin{aligned}
& \operatorname{abs}(\mathcal{G}, H)\left(\mathcal{M}^{\#}\right) \\
& \equiv \alpha_{\mathcal{G}} \circ f_{H} \circ \gamma_{\mathcal{G}}\left(\mathcal{M}^{\#}\right) \leq \mathcal{M}^{\#} \wedge \mathcal{M}^{\#} \leq \alpha_{\mathcal{G}}\left(\mathcal{U}_{H}\right)
\end{aligned}
$$

We use the item ii of Definition 14

$$
\equiv f_{H} \circ \gamma_{\mathcal{G}}\left(\mathcal{M}^{\#}\right) \leq \gamma_{\mathcal{G}}\left(\mathcal{M}^{\#}\right) \wedge \gamma_{\mathcal{G}}\left(\mathcal{M}^{\#}\right) \leq \mathcal{U}_{H}
$$

We use the item 5 of Theorem 13

$$
\begin{aligned}
\equiv f_{H} \circ \gamma_{\mathcal{G}} \circ \alpha_{\mathcal{G}} \circ \gamma_{\mathcal{G}}\left(\mathcal{M}^{\#}\right) \leq \gamma_{\mathcal{G}} \circ \alpha_{\mathcal{G}} \circ \gamma_{\mathcal{G}}\left(\mathcal{M}^{\#}\right) & \\
& \wedge \gamma_{\mathcal{G}} \circ \alpha_{\mathcal{G}} \circ \gamma_{\mathcal{G}}\left(\mathcal{M}^{\#}\right) \leq \mathcal{U}_{H}
\end{aligned}
$$

We use the item ii of Definition 14

$$
\begin{aligned}
& \equiv \alpha_{\mathcal{G}} \circ f_{H} \circ \gamma_{\mathcal{G}}\left(\alpha_{\mathcal{G}} \circ \gamma_{\mathcal{G}}\left(\mathcal{M}^{\#}\right)\right) \leq \alpha_{\mathcal{G}} \circ \gamma_{\mathcal{G}}\left(\mathcal{M}^{\#}\right) \wedge \\
& \alpha_{\mathcal{G}} \circ \gamma_{\mathcal{G}}\left(\mathcal{M}^{\#}\right) \leq \alpha_{\mathcal{G}}\left(\mathcal{U}_{H}\right) \\
& \equiv a b s(\mathcal{G}, H)\left(\alpha_{\mathcal{G}} \circ \gamma_{\mathcal{G}}\left(\mathcal{M}^{\#}\right)\right)
\end{aligned}
$$


Theorem 4 (Soundness with relative completeness is abs). If a transformation alg is sound and complete relative to $\mathcal{G}$, then

$$
\forall \mathfrak{C}, H_{\text {alg }(\mathfrak{C})} \text { satisfiable } \equiv \operatorname{abs}\left(\mathcal{G}, H_{\mathfrak{C}}\right) \text { satisfiable }
$$

In other words, when a transformation is sound and complete relative to an abstraction, it exactly does the abstraction.

Proof.

$$
H_{\mathfrak{C}} \text { satisfiable } \Rightarrow \operatorname{abs}\left(\mathcal{G}, H_{\mathfrak{C}}\right) \text { satisfiable }
$$

using Theorem 3, which combined with soundness yields

$$
H_{a l g(\mathfrak{C})} \text { satisfiable } \Rightarrow H_{\mathfrak{C}} \text { satisfiable }
$$

which used with relative completeness gives the equivalence.

Theorem 5 (Algorithm 1 is correct). If $P^{\#}$ is not used in $\mathfrak{C}$, then for any $\mathcal{M}^{\#}$,

$$
\left.H_{\text {dataabs }(\mathfrak{C}, P, P}, F_{\sigma}\right)\left(\mathcal{M}^{\#}\left[P^{\#} \leftarrow P\right]\right)=\operatorname{abs}\left(\mathcal{G}_{\sigma}^{P}, H_{\mathfrak{C}}\right)\left(\mathcal{M}^{\#}\right)
$$

Proof. $H_{\text {dataabs }\left(\mathfrak{C}, P, P \#, F_{\sigma}\right)}\left(\mathcal{M}^{\#}\left[P^{\#} \leftarrow P\right]\right)$

$={ }_{1} \bigwedge_{C \# \in \operatorname{dataabs}\left(\mathfrak{C}, P, P \#, F_{\sigma}\right)}^{\wedge} \llbracket$ vars,$C^{\#}($ vars $) \rrbracket_{\mathcal{M}^{\#}\left[P^{\#} \leftarrow P\right]}$

$=2 \bigwedge_{C \in \mathfrak{C}} \llbracket \forall$ vars,$C($ vars $) \rrbracket_{\gamma_{\mathcal{G}_{P}}(\mathcal{M} \#)}$

$={ }_{3} H_{\mathfrak{C}} \circ \gamma_{\mathcal{G}_{P}}\left(\mathcal{M}^{\#}\right)$

$={ }_{4} a b s\left(\mathcal{G}_{\sigma}^{P}, H_{\mathfrak{C}}\right)\left(\mathcal{M}^{\#}\right)$

The first and third equalities correspond to unrolling the definition of $H(\mathcal{M})$. The fourth equality is proved by Theorem 3 , now let us prove the second equality. Let $\operatorname{replaced}(e)=_{\text {def }} e$ where each instance of $P($ expr $)$ has been replaced by $\forall a^{\#}, F_{\sigma}\left(a^{\#}\right.$, expr $) \rightarrow P^{\#}\left(a^{\#}\right)$. Let us prove the second equality.

$$
\bigwedge_{C \# \in \operatorname{dataabs}\left(\mathfrak{C}, P, P^{\#}, F_{\sigma}\right)} \llbracket \forall \text { vars }, C^{\#}(\text { vars }) \rrbracket_{\mathcal{M} \#\left[P^{\#} \leftarrow P\right]}
$$

$=\bigwedge_{C \in \mathfrak{C}} \llbracket \forall$ vars, replaced $(C)$ (vars $) \rrbracket_{\mathcal{M}^{\#}\left[P^{\#} \leftarrow P\right]}$ by the definition of replaced.

We now prove equality 2 by proving that $\forall C \in \mathfrak{C}, \forall$ vars, $\llbracket$ replaced $(C)($ vars $) \rrbracket_{\mathcal{M}^{\#}[P \# \leftarrow P]}=\llbracket C($ vars $) \rrbracket_{\gamma_{\mathcal{G}_{P}}\left(\mathcal{M}^{\#}\right)}$.

We show it by showing, by structural induction on the expression $e$, the property $\operatorname{Ind}(e): \llbracket \operatorname{replaced}(e) \rrbracket_{\mathcal{M}^{\#}\left[P^{\#} \leftarrow P\right]}=\llbracket e \rrbracket_{\gamma_{\mathcal{G}_{P}}\left(\mathcal{M}^{\#}\right)}$.

Assume $e$ is built as Cons(exprs) using the Cons term constructor at the root. 1. if Cons is not a predicate (a boolean operator, for instance) then by the induction hypothesis, the evaluation of exprs is unchanged, thus $\operatorname{Ind}(e)$

2. if Cons is a predicate $P^{\prime}$ different from $P$, then $\gamma_{\mathcal{G}_{P}}$ does not affect $P^{\prime}$ and, by the induction hypothesis, the evaluation of exprs is unchanged, thus $\operatorname{Ind}(e)$

3 . if Cons is $P$, then $\llbracket$ replaced $(e) \rrbracket_{\mathcal{M} \#[P \# \leftarrow P]}$

$$
\begin{aligned}
& =\llbracket \forall a^{\#}, F_{\sigma}\left(a^{\#}, \text { exprs }\right) \rightarrow P^{\#}\left(a^{\#}\right) \rrbracket_{\mathcal{M} \#\left[P^{\#} \leftarrow P\right]} \\
& =\forall a^{\#}, F_{\sigma}\left(a^{\#}, \text { exprs }\right) \rightarrow \mathcal{M}^{\#}\left[P^{\#} \leftarrow P\right]\left(P^{\#}\right)\left(a^{\#}\right)
\end{aligned}
$$




$$
\begin{aligned}
& =\forall a^{\#}, F_{\sigma}\left(a^{\#}, \text { exprs }\right) \rightarrow \mathcal{M}^{\#}(P)\left(a^{\#}\right) \\
& =\operatorname{exprs} \in\left\{c \mid \sigma(c) \subseteq \mathcal{M}^{\#}(P)\right\} \\
& =\operatorname{exprs} \in \gamma_{\sigma}\left(\mathcal{M}^{\#}(P)\right) \\
& =\operatorname{exprs} \in \gamma_{\mathcal{G}_{P}}\left(\mathcal{M}^{\#}\right)(P) \\
& =\llbracket P(\text { exprs }) \rrbracket_{{\mathcal{G}_{P}}_{\mathcal{G}}\left(\mathcal{M}^{\#}\right)}
\end{aligned}
$$

Thus, $\operatorname{Ind}(e)$ holds in all cases.

\section{Theorem 6 (Soundness of eliminate).}

$$
\forall C, \text { insts, } \mathcal{M}, \llbracket \text { eliminate }(C, \text { insts }) \rrbracket_{\mathcal{M}} \Rightarrow \llbracket C \rrbracket_{\mathcal{M}}
$$

Proof. Fix $C$, insts, $\mathcal{M}$. We have $\forall i, \llbracket e_{i} \rrbracket_{\mathcal{M}} \Rightarrow \llbracket e_{\text {res }} \rrbracket_{\mathcal{M}}$ as the finite conjunction over $I_{i}$ in $e_{i}$ is contained in the infinite conjunction of $e_{i}$. Then $\llbracket e_{1} \wedge$ $\ldots \wedge e_{n} \rrbracket_{\mathcal{M}} \Rightarrow \llbracket e_{r e s_{1}} \wedge \ldots \wedge e_{r_{e s}} \rrbracket_{\mathcal{M}}$. Finally, as $\llbracket e^{\prime} \rrbracket_{\mathcal{M}} \equiv \llbracket e_{\text {res }}^{\prime} \rrbracket_{\mathcal{M}}$, we have $\llbracket$ eliminate $(C$, insts $) \rrbracket_{\mathcal{M}} \Rightarrow \llbracket C \rrbracket_{\mathcal{M}}$.

\section{A.2 Completeness and strong complenetess}

Theorem 7 (Completeness of calls to insts implies completeness of eliminate).

For any $C$, insts, $\mathcal{M}$, if during execution of eliminate $(C$, insts) all calls to insts are complete, then

$$
\llbracket C \rrbracket_{\alpha_{\mathcal{G}}(\mathcal{M})}=\llbracket \text { eliminate }(C, \text { insts }) \rrbracket_{\alpha_{\mathcal{G}}(\mathcal{M})}
$$

where $\mathcal{G}$ is such that $\forall i, \gamma_{\mathcal{G}}\left(\mathcal{M}\left(P_{i}^{\#}\right)\right)=\gamma_{\sigma_{i}}\left(P_{i}^{\#}\right)$, with $i, P_{i}^{\#}, \sigma_{i}$ as defined in eliminate.

Proof. Let $C_{i}=e_{\text {res }} \wedge \ldots \wedge e_{\text {res }_{i-1}} \wedge e_{\text {res }_{i}} \wedge e_{i+1} \ldots \wedge e_{n} \rightarrow e_{\text {res }}^{\prime}$. and let $\mathcal{M}^{\prime}=$ $\alpha_{\mathcal{G}}(\mathcal{M})$. Our algorithm eliminate takes as input a clause equivalent to $C_{0}$ as step 1 does not change the semantics, and outputs $C_{n}$. Let us show by induction on $i$ that $\llbracket C_{i} \rrbracket_{\mathcal{M}^{\prime}} \equiv \llbracket C_{0} \rrbracket_{\mathcal{M}^{\prime}}$. 


$$
\begin{aligned}
& \llbracket C_{i+1} \rrbracket_{\mathcal{M}^{\prime}} \\
& \equiv \llbracket e_{r e s_{i+1}} \rightarrow e_{r e s_{1}} \wedge \ldots \wedge e_{r e s_{i-1}} \wedge e_{r e s_{i}} \wedge e_{i+2} \wedge \ldots \wedge e_{n} \rightarrow e_{r e s}^{\prime} \rrbracket_{\mathcal{M}^{\prime}} \\
& \equiv \llbracket e_{r e s_{i+1}} \rightarrow c t x_{i+1} \rrbracket_{\mathcal{M}^{\prime}} \\
& \equiv \forall \operatorname{vars},\left(\forall\left(\left(a^{\#}, q\right)\right) \in \operatorname{insts}\left(F_{\sigma_{i+1}}, a_{i+1}, c t x_{i+1}\right)(\text { vars }),\right. \\
& \left.F_{\sigma_{i+1}}[q]\left(a^{\#}, a_{i+1}(\text { vars })\right) \Rightarrow a^{\#} \in \mathcal{M}^{\prime}\left(P_{i+1}^{\#}\right)\right) \rightarrow \llbracket c t x_{i+1}(\text { vars }) \rrbracket_{\mathcal{M}^{\prime}} \\
& \text { expanding the match pattern } \\
& \equiv \forall \operatorname{vars},\left(\forall\left(\left(a^{\#}, q\right)\right), F_{\sigma_{i+1}}[q]\left(a^{\#}, a_{i+1}(\text { vars })\right)\right. \\
& \left.\Rightarrow a^{\#} \in \mathcal{M}^{\prime}\left(P_{i+1}^{\#}\right)\right) \rightarrow \llbracket c t x_{i+1}(\text { vars }) \rrbracket_{\mathcal{M}^{\prime}} \\
& \text { using completeness of that call to insts, with } \alpha_{\sigma}(E)=\mathcal{M}^{\prime}\left(P_{i+1}^{\#}\right) \\
& \equiv \llbracket e_{i+1} \rightarrow e_{r e s_{1}} \wedge \ldots \wedge e_{r e s_{i-1}} \wedge e_{r e s_{i}} \wedge e_{i+2} \wedge \ldots \wedge e_{n} \rightarrow e_{r e s}^{\prime} \rrbracket_{\mathcal{M}^{\prime}} \\
& \text { using the match pattern } \\
& \equiv \llbracket C_{i} \rrbracket_{\mathcal{M}^{\prime}} \text { decurrying } \\
& \equiv \llbracket C_{0} \rrbracket_{\mathcal{M}^{\prime}} \text { by induction assumption }
\end{aligned}
$$

Theorem 8 (Strong completeness implies completeness). If ctx is of boolean type, insts $\left(F_{\sigma}, a, c t x\right)$ strongly complete $\Rightarrow$ insts $\left(F_{\sigma}, a\right.$, ctx $)$ complete

Proof. Assume strong completeness of insts $\left(F_{\sigma}, a, c t x\right)$ and fix $E, \mathcal{M}$. By contraposition, completeness of insts $\left(F_{\sigma}, a, c t x\right)$ is equivalent to :

$$
\begin{gathered}
\left(\exists \text { vars }, \neg \llbracket \operatorname{ctx}(\text { vars }) \rrbracket_{\mathcal{M}} \wedge\right. \\
F_{\sigma}[q]\left(a^{\#}, a\left(\left(a^{\#}, q\right)\right) \in \operatorname{insts}\left(F_{\sigma}, a, c t x\right)\right)(\text { vars }), \\
\left.\left.\Rightarrow a^{\#} \in \alpha_{\sigma}(E)\right)\right) \\
\quad\left(\exists \operatorname{vars}^{\prime}, \neg \llbracket \operatorname{ct} x\left(\text { vars }^{\prime}\right) \rrbracket_{\mathcal{M}} \wedge\right. \\
\left.\left(\forall\left(\left(a^{\#}, q\right)\right), F_{\sigma}[q]\left(a^{\#}, a(\text { vars })\right) \Rightarrow a^{\#} \in \alpha_{\sigma}(E)\right)\right)
\end{gathered}
$$

Now introduce vars and assume the first part of the implication.

Therefore we have, $\forall\left(\left(a^{\#}, q\right)\right) \in \operatorname{insts}\left(F_{\sigma}, a, c t x\right)($ vars $), F_{\sigma}[q]\left(a^{\#}, a(\right.$ vars $\left.)\right) \Rightarrow a^{\#} \in \alpha_{\sigma}(E)$, and we obtain using strong completeness

$$
\begin{aligned}
\exists \operatorname{vars}^{\prime},\left(\forall\left(a^{\#}, q\right), F_{\sigma}[q]\left(a^{\#}, a(\text { vars })\right) \Rightarrow a^{\#}\right. & \in \alpha_{\sigma}(E) \wedge \\
& \llbracket c t x(\text { vars }) \rrbracket_{\mathcal{M}}=\llbracket c t x\left(\text { vars }^{\prime}\right) \rrbracket_{\mathcal{M}} .
\end{aligned}
$$

But using $\neg \llbracket \operatorname{ctx}($ vars $) \rrbracket_{\mathcal{M}}$ (assumed in the first part of completeness), and $\llbracket \operatorname{ctx}($ vars $) \rrbracket_{\mathcal{M}}=\llbracket \operatorname{ctx}\left(\right.$ vars $\left.^{\prime}\right) \rrbracket_{\mathcal{M}}$ we obtain $\neg \llbracket \operatorname{ctx}\left(\right.$ vars $\left.^{\prime}\right) \rrbracket_{\mathcal{M}}$.

Therefore, we have both elements of our contraposed completeness and our proof is complete. 
Even though most abstractions for unbounded data structures are fairly complex and involve infinitely many abstract values for some concrete values (i.e. cardinal $(\sigma(a))$ is infinite) as is the case for $\mathrm{Cell}_{1}$, many "filler" abstractions such as $\sigma_{i d}$, or simpler abstractions (see Section 5) are finite. For finite abstractions, one can define a very simple heuristic insts which verifies the strong completeness property : return the set of abstract elements!

Theorem 9 (Strong Completeness of insts of Algorithms 3 and 4 ).

If $\sigma$ is finite, insts $\left(F_{\sigma}, a\right.$, ctx $)$ is strongly complete. If its recursive calls are strongly complete, insts $\left(F_{\sigma_{1} \cdot \sigma_{2}},\left(a_{1}, a_{2}\right)\right.$, ctx $)$ is strongly complete. If $\sigma_{1}, \sigma_{2}$ are compatible: $\forall E \neq \emptyset, \alpha_{\sigma_{2}} \circ \gamma_{\sigma_{2}} \circ \gamma_{\sigma_{1}} \circ \alpha_{\sigma_{1}} \circ \alpha_{\sigma_{2}}(E)=\gamma_{\sigma_{1}} \circ \alpha_{\sigma_{1}} \circ \alpha_{\sigma_{2}}(E)$ and its recursive calls are strongly complete, then insts $\left(F_{\sigma_{1} \circ \sigma_{2}}, a, c t x\right)$ is strongly complete.

Proof. - finite case

Assume $F_{\sigma}$ does not use nested existential quantifiers and

$\forall$ vars, $\sigma(a($ vars $)) \subseteq$ insts $\left(F_{\sigma}, a, c t x\right)($ vars $)$ and introduce $E$, vars, $\mathcal{M}$.

$$
\begin{aligned}
& \forall\left(\left(a^{\#},()\right)\right) \in \operatorname{insts}\left(F_{\sigma}, a, c t x\right)(\text { vars }), \\
& F_{\sigma}[()]\left(a^{\#}, a(\text { vars })\right) \Rightarrow a^{\#} \in \alpha_{\sigma}(E) \\
\equiv & \forall\left(\left(a^{\#},()\right)\right) \in \operatorname{insts}\left(F_{\sigma}, a, c t x\right)(\text { var }) \cap \sigma(a(\text { vars })), a^{\#} \in \alpha_{\sigma}(E) \\
\equiv & \forall\left(\left(a^{\#},()\right)\right) \in \sigma(a(\text { vars })), a^{\#} \in \alpha_{\sigma}(E) \text { by assumption } \\
\equiv & \left(\forall\left(a^{\#},()\right), F_{\sigma}[()]\left(a^{\#}, a\left(\text { vars }^{\prime}\right)\right) \Rightarrow a^{\#} \in \alpha_{\sigma}(E)\right) \\
\wedge & \llbracket \operatorname{ctx}(\text { vars }) \rrbracket_{\mathcal{M}}=\llbracket \operatorname{ctx}\left(\text { vars }^{\prime}\right) \rrbracket \text { with } \text { vars }^{\prime}=\text { vars }
\end{aligned}
$$

As a consequence, we have:

- $\forall a, c t x$, insts $\left(F_{\sigma_{i d}}, a, c t x\right)$ as defined in Algorithm 3 is strongly complete.

- $\forall a$, ctx , insts $\left(F_{\sigma_{b o t}}, a, c t x\right)$ as defined in Algorithm 3 is strongly complete.

- inductive cases: for dot and o: see below

Theorem 9, Strong completeness for .

$\forall \sigma_{1}, \sigma_{2}, a_{1}, a_{2}$, ctx, insts $\left(F_{\sigma_{1} \cdot \sigma_{2}},\left(a_{1}, a_{2}\right), c t x\right)$ as defined in Algorithm 4 is strongly complete when its recursive calls to insts, that is, insts $\left(F_{\sigma_{1}}, a_{1},\left(c t x, a_{2}\right)\right)$ and $\operatorname{insts}\left(F_{\sigma_{2}}, a_{2},\left(\right.\right.$ ctx $\left.\left., a_{1}, I_{1}\right)\right)$ are.

Proof. For insts $\left(F_{\sigma_{1} \cdot \sigma_{2}},\left(a_{1}, a_{2}\right), c t x\right)$, the idea consists in first abstracting $a_{2}$ (respectively $a_{1}$ ) thus yielding a vars $s_{t m p}$ and then abstracting $a_{1}$ yielding a vars'. The key thing is that when abstracting $a_{2}$, we must not change anything to the rest of the clause, including the instantiation sets. Now, let us do the full proof of strong completeness by working from the assumption we have in strong completeness:

$$
\begin{aligned}
& \forall\left(a^{\#}, q\right) \in \operatorname{insts}\left(F_{\sigma_{1} \cdot \sigma_{2}}, a, c t x\right)(\text { vars }), \\
& \quad F_{\sigma_{1} \cdot \sigma_{2}}[q]\left(a^{\#}, a(\text { vars })\right) \Rightarrow a^{\#} \in \alpha_{\sigma_{1} \cdot \sigma_{2}}(E)
\end{aligned}
$$


1) Let us unpack $a=\left(a_{1}, a_{2}\right)$ and $q=q_{1} @ q_{2}$ As we know the number of identifiers generated by $\sigma_{1}$, we know where to split the list $q$

$$
\begin{aligned}
& \forall\left(\left(a_{1}^{\#}, a_{2}^{\#}\right), q_{1} @ q_{2}\right) \in \operatorname{insts}\left(F_{\sigma_{1} \cdot \sigma_{2}},\left(a_{1}, a_{2}\right), \text { ctx }\right)(\text { vars }), \\
& \qquad \begin{array}{l}
F_{\sigma_{1} \cdot \sigma_{2}}\left[q_{1} @ q_{2}\right]\left(\left(a_{1}^{\#}, a_{2}^{\#}\right),\left(a_{1}(\text { vars }), a_{2}(\text { vars })\right)\right) \\
\Rightarrow\left(a_{1}^{\#}, a_{2}^{\#}\right) \in \alpha_{\sigma_{1} \cdot \sigma_{2}}(E)
\end{array}
\end{aligned}
$$

2) Using the definition of insts $\left(F_{\sigma_{1} \cdot \sigma_{2}},\left(a_{1}, a_{2}\right)\right.$, ctx $), I_{1}, I_{2}$

$$
\begin{aligned}
& \forall\left(a_{1}^{\#}, q_{1}\right) \in I_{1}(\text { vars }),\left(a_{2}^{\#}, q_{2}\right) \in I_{2}(\text { vars }), \\
& \qquad \begin{array}{l}
F_{\sigma_{1} \cdot \sigma_{2}}\left[q_{1} @ q_{2}\right]\left(\left(a_{1}^{\#}, a_{2}^{\#}\right),\left(a_{1}(\text { vars }), a_{2}(\text { vars })\right)\right) \\
\Rightarrow\left(a_{1}^{\#}, a_{2}^{\#}\right) \in \alpha_{\sigma_{1} \cdot \sigma_{2}}(E)
\end{array}
\end{aligned}
$$

3) Using the definition of $F_{\sigma_{1}, \sigma_{2}}$

$$
\begin{aligned}
\forall\left(a_{1}^{\#}, q_{1}\right) \in I_{1}(\text { vars }),\left(a_{2}^{\#}, q_{2}\right) \in & I_{2}(\text { vars }), F_{\sigma_{1}}\left[q_{1}\right]\left(a_{1}^{\#}, a_{1}(\text { vars })\right) \\
& \wedge F_{\sigma_{2}}\left[q_{2}\right]\left(a_{2}^{\#}, a_{2}(\text { vars })\right) \Rightarrow\left(a_{1}^{\#}, a_{2}^{\#}\right) \in \alpha_{\sigma_{1} \cdot \sigma_{2}}(E)
\end{aligned}
$$

4) Now, let us move things around

$$
\begin{aligned}
& \forall\left(a_{2}^{\#}, q_{2}\right) \in I_{2}(\text { vars }), F_{\sigma_{2}}\left[q_{2}\right]\left(a_{2}^{\#}, a_{2}(\text { vars })\right) \\
& \Rightarrow \forall\left(a_{1}^{\#}, q_{1}\right) \in I_{1}(\text { vars }), F_{\sigma_{1}}\left[q_{1}\right]\left(a_{1}^{\#}, a_{1}(\text { vars })\right) \\
& \quad \Rightarrow\left(a_{1}^{\#}, a_{2}^{\#}\right) \in \alpha_{\sigma_{1} \cdot \sigma_{2}}(E)
\end{aligned}
$$

5) Now, let us prepare to use strong completeness of $I_{2}$

$$
\begin{aligned}
\forall\left(a_{1}^{\#}, q_{1}\right) \in I_{1}(\text { vars }) & , F_{\sigma_{1}}\left[q_{1}\right]\left(a_{1}^{\#}, a_{1}(\text { vars })\right) \\
\Rightarrow & \forall\left(a_{2}^{\#}, q_{2}\right) \in I_{2}(\text { vars }), F_{\sigma_{2}}\left[q_{2}\right]\left(a_{2}^{\#}, a_{2}(\text { vars })\right) \\
& \Rightarrow a_{2}^{\#} \in\left\{x \mid\left(a_{1}^{\#}, x\right) \in \alpha_{\sigma_{1} \cdot \sigma_{2}}(E)\right\}
\end{aligned}
$$

6) The set in question is a projection. Using the definition of $\alpha_{\sigma_{1} \cdot \sigma_{2}}$, we have :

$$
\begin{aligned}
& \forall\left(a_{1}^{\#}, q_{1}\right) \in I_{1}(\text { vars }), F_{\sigma_{1}}\left[q_{1}\right]\left(a_{1}^{\#}, a_{1}(\text { vars })\right) \\
& \Rightarrow \forall\left(a_{2}^{\#}, q_{2}\right) \in I_{2}(\text { vars }), \\
& \quad F_{\sigma_{2}}\left[q_{2}\right]\left(a_{2}^{\#}, a_{2}(\text { vars })\right) \\
& \quad \Rightarrow a_{2}^{\#} \in \alpha_{\sigma_{2}}\left(\left\{x \mid\left(a_{1}^{\#}, x\right) \in \alpha_{\sigma_{1} \cdot \sigma_{i d}}(E)\right\}\right)
\end{aligned}
$$

7) Let $E_{t}$ be the latter set, yielding

$$
\begin{aligned}
\forall\left(a_{1}^{\#}, q_{1}\right) \in I_{1}(\text { vars }) & , F_{\sigma_{1}}\left[q_{1}\right]\left(a_{1}^{\#}, a_{1}(\text { vars })\right) \\
\Rightarrow & \forall\left(a_{2}^{\#}, q_{2}\right) \in I_{2}(\text { vars }), F_{\sigma_{2}}\left[q_{2}\right]\left(a_{2}^{\#}, a_{2}(\text { vars })\right) \Rightarrow a_{2}^{\#} \in \alpha_{\sigma_{2}}\left(E_{t}\right)
\end{aligned}
$$

8) Let us move the first implication to the context.

$$
\forall\left(a_{2}^{\#}, q_{2}\right) \quad \in \quad I_{2}(\text { vars }), F_{\sigma_{2}}\left[q_{2}\right]\left(a_{2}^{\#}, a_{2}(\text { vars })\right) \quad \Rightarrow \quad a_{2}^{\#} \quad \in \quad \alpha_{\sigma_{2}}\left(E_{t}\right)
$$


9) Let use strong completeness of $I_{2}=\operatorname{insts}\left(F_{\sigma_{2}}, a_{2},\left(c t x, a_{1}, I_{1}\right)\right)$ with $E=E_{t}$ We retrieve vars $_{t}$ such that

$$
\begin{aligned}
\left(\forall\left(a_{2}^{\#}, q_{2}\right), F_{\sigma_{2}}\left[q_{2}\right]\right. & \left.\left(a_{2}^{\#}, a_{2}\left(\text { vars }_{t}\right)\right) \Rightarrow a_{2}^{\#} \in \alpha_{\sigma_{2}}\left(E_{t}\right)\right) \\
& \wedge \llbracket \operatorname{ctx}(\text { vars }) \rrbracket_{\mathcal{M}}=\llbracket \operatorname{ctx}\left(\text { vars }_{t}\right) \rrbracket_{\mathcal{M}} \\
\wedge \llbracket a_{1}(\text { vars }) \rrbracket_{\mathcal{M}}=\llbracket a_{1}\left(\text { var }_{t}\right) \rrbracket_{\mathcal{M}} & \wedge \llbracket I_{1}(\text { vars }) \rrbracket_{\mathcal{M}}=\llbracket I_{1}\left(\text { vars }_{t}\right) \rrbracket_{\mathcal{M}}
\end{aligned}
$$

10) Let us unfold $E_{t}$ using the equalities and reintroducing the implication of step 8

$$
\begin{aligned}
& \left(\forall\left(a_{2}^{\#}, q_{2}\right), F_{\sigma_{2}}\left[q_{2}\right]\left(a_{2}^{\#}, a_{2}\left(\operatorname{vars}_{t}\right)\right) \Rightarrow\right. \\
& \forall\left(a_{1}^{\#}, q_{1}\right) \in I_{1}\left(\text { vars }_{t}\right), F_{\sigma_{1}}\left[q_{1}\right]\left(a_{1}^{\#}, a_{1}\left(\operatorname{vars}_{t}\right)\right) \\
& \quad \Rightarrow\left(a_{1}^{\#}, a_{2}^{\#}\right) \in \alpha_{\sigma_{1} \cdot \sigma_{2}}(E)
\end{aligned}
$$

11) We remember $\llbracket c t x($ vars $) \rrbracket_{\mathcal{M}}=\llbracket c t x\left(\right.$ var $\left._{t}\right) \rrbracket_{\mathcal{M}}$

Now that we have removed $I_{2}$, let us repeat steps after 4 for $I_{1}$

$$
\begin{aligned}
& \forall\left(a_{2}^{\#}, q_{2}\right), F_{\sigma_{2}}\left[q_{2}\right]\left(a_{2}^{\#}, a_{2}\left(\text { vars }_{t}\right)\right) \\
& \quad \Rightarrow \forall\left(a_{1}^{\#}, q_{1}\right) \in I_{1}\left(\text { vars }_{t}\right), F_{\sigma_{1}}\left[q_{1}\right]\left(a_{1}^{\#}, a_{1}\left(\text { vars }_{t}\right)\right) \\
& \quad \Rightarrow a_{1}^{\#} \in \alpha_{\sigma_{1}}\left(\left\{x \mid\left(x, a_{2}^{\#}\right) \in \alpha_{i d \cdot \sigma_{2}}(E)\right\}\right)
\end{aligned}
$$

12) Let us name that set $E_{t}^{\prime}$ and let use strong completeness of $I_{1}$ (moving the first implication in context)

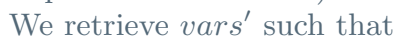

$$
\begin{aligned}
&\left(\forall\left(a_{1}^{\#}, q_{1}\right), F_{\sigma_{1}}\left[q_{1}\right]\left(a_{1}^{\#}, a_{1}\left(\text { vars }^{\prime}\right)\right) \Rightarrow a_{1}^{\#} \in \alpha_{\sigma_{1}}\left(E_{t}^{\prime}\right)\right) \\
& \wedge \llbracket \operatorname{ctx}\left(\text { vars }_{t}\right) \rrbracket_{\mathcal{M}}=\llbracket \operatorname{ctx}\left(\text { vars }^{\prime}\right) \rrbracket_{\mathcal{M}} \\
& \wedge \llbracket a_{2}\left(\text { vars }_{t}\right) \rrbracket_{\mathcal{M}}=\llbracket a_{2}\left(\text { vars }^{\prime}\right) \rrbracket_{\mathcal{M}}
\end{aligned}
$$

13) Let us unfold $E_{t}^{\prime}$ using the equalities and context

$$
\begin{aligned}
& \forall\left(a_{1}^{\#}, q_{1}\right), F_{\sigma_{1}}\left[q_{1}\right]\left(a_{1}^{\#}, a_{1}\left(v \operatorname{vrs}^{\prime}\right)\right) \\
& \quad \Rightarrow \forall\left(a_{2}^{\#}, q_{2}\right), F_{\sigma_{2}}\left[q_{2}\right]\left(a_{2}^{\#}, a_{2}\left(v_{a r s^{\prime}}\right)\right) \Rightarrow\left(a_{1}^{\#}, a_{2}^{\#}\right) \in \alpha_{\sigma_{1} \cdot \sigma_{2}}(E)
\end{aligned}
$$

14) Reversing the steps before 4 and using $\llbracket c t x($ vars $) \rrbracket_{\mathcal{M}}=\llbracket c t x\left(\right.$ vars $\left._{t}\right) \rrbracket_{\mathcal{M}}=\llbracket c t x\left(\right.$ vars $\left.^{\prime}\right) \rrbracket_{\mathcal{M}}($ steps 11 and 12

$$
\begin{aligned}
\left(\forall\left(a^{\#}, q\right), F_{\sigma_{1} \cdot \sigma_{2}}[q]\left(a^{\#}, a\left(\text { vars }^{\prime}\right)\right) \Rightarrow a^{\#} \in \alpha_{\sigma_{1} \cdot \sigma_{2}}(E)\right) & \wedge \llbracket \operatorname{ctx}(\text { vars }) \rrbracket_{\mathcal{M}}=\llbracket c t x\left(\text { vars }^{\prime}\right) \rrbracket_{\mathcal{M}}
\end{aligned}
$$

15) Our desired result !

\section{Theorem 9 (Strong completeness for $\circ$ ).}

$\forall \sigma_{1}, \sigma_{2}, a$, ctx, insts $\left(F_{\sigma_{1} \circ \sigma_{2}}, a\right.$, ctx $)$ as defined in Algorithm 4 is strongly complete when

- its recursive calls to insts, that is, $I_{2},\left\{I_{\left(q_{0}, q_{2}\right)},\left(q_{0}, q_{2}\right) \in I_{2}\right\}$ are.

$-\sigma_{1}, \sigma_{2}$ are compatible, that is :

$\forall E \neq \emptyset, \alpha_{\sigma_{2}} \circ \gamma_{\sigma_{2}} \circ \gamma_{\sigma_{1}} \circ \alpha_{\sigma_{1}} \circ \alpha_{\sigma_{2}}(E)=\gamma_{\sigma_{1}} \circ \alpha_{\sigma_{1}} \circ \alpha_{\sigma_{2}}(E)$ 
Proof. The case $E=\emptyset$ is proven by definition of string completeness. Let us continue with $E \neq \emptyset$.

$$
\begin{aligned}
& \forall\left(a^{\#}, q\right) \in \operatorname{insts}\left(F_{\sigma_{1} \circ \sigma_{2}}, a, c t x\right)(\text { vars }), \\
& \qquad F_{\sigma_{1} \circ \sigma_{2}}[q]\left(a^{\#}, a(\text { vars })\right) \Rightarrow a^{\#} \in \alpha_{\sigma_{1} \circ \sigma_{2}}(E)
\end{aligned}
$$

16) Unfolding definition of insts $\left(F_{\sigma_{1} \circ \sigma_{2}}, a, c t x\right)$ (using the first note) and $F_{\sigma_{1} \circ \sigma_{2}}$. We remind that $\left(a^{\#},\left(q_{0} @ q_{1} @ q_{2}\right)\right) \in \operatorname{insts}\left(F_{\sigma_{1} \circ \sigma_{2}}, a, c t x\right) \equiv\left(q_{0}, q_{2}\right) \in I_{2} \wedge\left(a^{\#}, q_{1}\right) \in I_{\left(q_{0}, q_{2}\right)}$

$$
\begin{aligned}
& \forall\left(q_{0}, q_{2}\right) \in I_{2}(\text { vars }), \forall\left(a^{\#}, q_{1}\right) \in I_{\left(q_{0}, q_{2}\right)}(\text { vars }), \\
& \quad F_{\sigma_{2}}\left[q_{2}\right]\left(q_{0}, a(\text { vars })\right) \wedge F_{\sigma_{1}}\left[q_{1}\right]\left(a^{\#}, q_{0}\right) \Rightarrow a^{\#} \in \alpha_{\sigma_{1} \circ \sigma_{2}}(E)
\end{aligned}
$$

17) We first wish to transform the universal quantification restricted to $I_{\left(q_{0}, q_{2}\right)}$ into an unrestricted one. As there is an instance of $I_{\left(q_{0}, q_{2}\right)}$ for each $\left(q_{0}, q_{2}\right) \in I_{2}$, we iteratively handle each instance. At each stage of the iteration we have the set of instances that have already been handled $I_{t m p}$, and the set that is still to handle $I_{2}-I_{t m p}$. This yields the following property Handled $\left(\boldsymbol{I}_{t m p}\right)$.

$\exists$ vars $^{\prime}$,
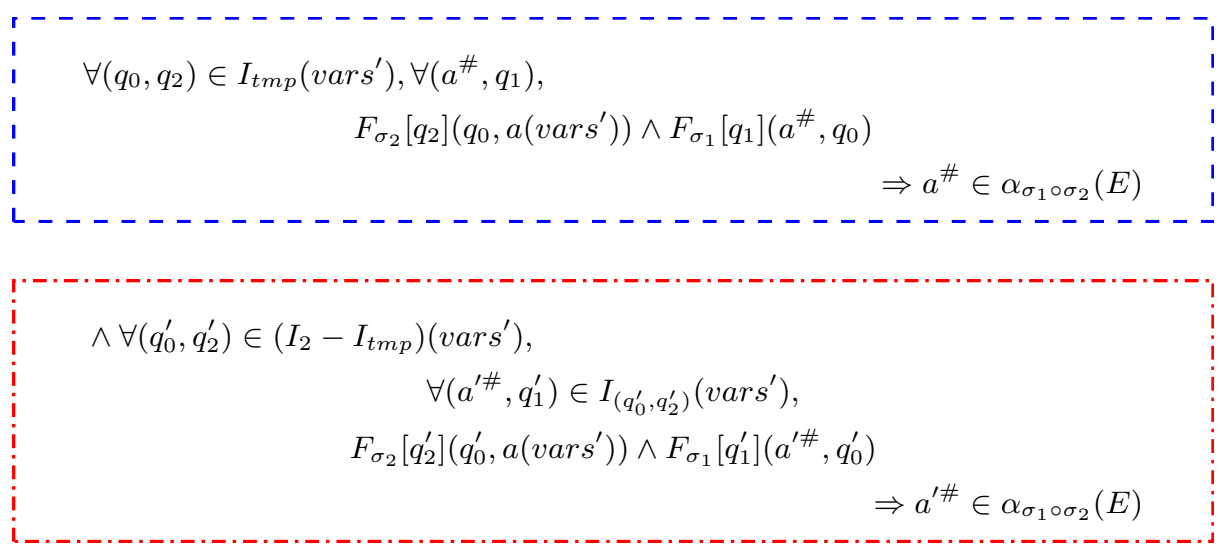

$\wedge \llbracket c t x\left(\operatorname{vars}^{\prime}\right) \rrbracket_{\mathcal{M}}=\llbracket c t x($ vars $) \rrbracket_{\mathcal{M}}$

Note that the property is divided in three parts

r. 1 - The handled part, where the quantifiers are unrestricted.

I_ - - - - - - - - - - - - - - - - - -

- The not yet handled part, where the quantifiers are restricted

- The rest

From Step 16 we have :

Handled $(\emptyset)$ 
18) The proof that Handled $(\emptyset) \Rightarrow \operatorname{Handled}\left(I_{2}\right)$ is done in step 25 Assuming it, we have

\section{$\operatorname{Handled}\left(I_{2}\right)$}

19) Unfolding the definition of $\operatorname{Handled}\left(I_{2}\right)$, thus retrieving a $\exists$ vars' and introducing it as varst, we get :

$$
\begin{aligned}
& \forall\left(q_{0}, q_{2}\right) \in I_{2}\left(\text { vars }_{t}\right), \forall\left(a^{\#}, q_{1}\right), \\
& F_{\sigma_{2}}\left[q_{2}\right]\left(q_{0}, a\left(\text { vars }_{t}\right)\right) \wedge \\
& \Rightarrow F_{\sigma_{1}}\left[q_{1}\right]\left(a^{\#}, q_{0}\right) \\
& \Rightarrow a^{\#} \in \alpha_{\sigma_{1} \circ \sigma_{2}}(E) \\
& \wedge \llbracket c t x(\text { vars }) \rrbracket_{\mathcal{M}}=\llbracket c t x\left(\text { vars }_{t}\right) \rrbracket_{\mathcal{M}}
\end{aligned}
$$

20) Let us prepare to apply strong completeness of $I_{2}$ and let us remember $\llbracket c t x($ vars $) \rrbracket_{\mathcal{M}}=$ $\llbracket c t x\left(\operatorname{var}_{t}\right) \rrbracket_{\mathcal{M}}$

$$
\begin{aligned}
& \forall\left(q_{0}, q_{2}\right) \in I_{2}\left(\operatorname{vars}_{t}\right), F_{\sigma_{2}}\left[q_{2}\right]\left(q_{0}, a\left(\operatorname{vars}_{t}\right)\right) \\
& \Rightarrow q_{0} \in\left\{x \mid \forall\left(a^{\#}, q_{1}\right), F_{\sigma_{1}}\left[q_{1}\right]\left(a^{\#}, x\right) \Rightarrow a^{\#} \in \alpha_{\sigma_{1} \circ \sigma_{2}}(E)\right\}
\end{aligned}
$$

21) Let us name the set $\left\{x \mid \forall\left(a^{\#}, q_{1}\right), F_{\sigma_{1}}\left[q_{1}\right]\left(a^{\#}, x\right) \Rightarrow a^{\#} \in \alpha_{\sigma_{1} \circ \sigma_{2}}(E)\right\}$ as $E_{I_{2}}$. To apply strong completeness of $I_{2}=\operatorname{insts}\left(F_{\sigma_{2}}, a, c t x\right)$, we need $\exists E, E_{I_{2}}=\alpha_{\sigma_{2}}(E)$. We show that $E_{I_{2}}=\alpha_{\sigma_{2}} \circ$ $\gamma_{\sigma_{2}}\left(E_{I_{2}}\right)$ and thus, our need is verified for $E=\gamma_{\sigma_{2}}\left(E_{I_{2}}\right)$.

$E_{I_{2}}=\left\{x \mid \forall\left(a^{\#}, q_{1}\right), F_{\sigma_{1}}\left[q_{1}\right]\left(a^{\#}, x\right) \Rightarrow a^{\#} \in \alpha_{\sigma_{1} \circ \sigma_{2}}(E)\right\}$

$=\left\{x \mid \forall a^{\#} \in \sigma_{1}(x), a^{\#} \in \alpha_{\sigma_{1}} \circ \alpha_{\sigma_{2}}(E)\right\}$

$=\left\{x \mid \sigma_{1}(x) \subseteq \alpha_{\sigma_{1}} \circ \alpha_{\sigma_{2}}(E)\right\}$

$=\bigcup\left\{z \mid \alpha_{\sigma_{1}}(z) \subseteq \alpha_{\sigma_{1}} \circ \alpha_{\sigma_{2}}(E)\right\}$

$=\bigcup\left\{z \mid z \subseteq \gamma_{\sigma_{1}} \circ \alpha_{\sigma_{1}} \circ \alpha_{\sigma_{2}}(E)\right\}$

$=\gamma_{\sigma_{1}} \circ \alpha_{\sigma_{1}} \circ \alpha_{\sigma_{2}}(E)$

Therefore, $\alpha_{\sigma_{2}} \circ \gamma_{\sigma_{2}}\left(E_{I_{2}}\right)$

$=\alpha_{\sigma_{2}} \circ \gamma_{\sigma_{2}} \circ \gamma_{\sigma_{1}} \circ \alpha_{\sigma_{1}} \circ \alpha_{\sigma_{2}}(E)$

$=\gamma_{\sigma_{1}} \circ \alpha_{\sigma_{1}} \circ \alpha_{\sigma_{2}}(E)$

by compatibility assumption

$=E_{I_{2}}$

Let us continue our main proof and let us replace the $E_{I_{2}}$ by $\alpha_{\sigma_{2}} \circ \gamma_{\sigma_{2}}\left(E_{I_{2}}\right)$

$\forall\left(q_{0}, q_{2}\right) \in I_{2}\left(\right.$ vars $\left._{t}\right), F_{\sigma_{2}}\left[q_{2}\right]\left(q_{0}, a\left(\right.\right.$ vars $\left.\left._{t}\right)\right)$

$$
\Rightarrow q_{0} \in \alpha_{\sigma_{2}} \circ \gamma_{\sigma_{2}}\left(E_{I_{2}}\right)
$$

$22)$ Let use strong completeness of $I_{2}=\operatorname{insts}\left(F_{\sigma_{2}}, a, c t x\right)$ with $E=\gamma_{\sigma_{2}}\left(E_{I_{2}}\right)$. We retrieve vars' such that (and reusing $E_{I_{2}}=\alpha_{\sigma_{2}} \circ \gamma_{\sigma_{2}}\left(E_{I_{2}}\right)$ and unfolding the definition of $E_{I_{2}}$

$$
\begin{aligned}
& \forall\left(q_{0}, q_{2}\right), F_{\sigma_{2}}\left[q_{2}\right]\left(q_{0}, a\left(\text { vars }^{\prime}\right)\right) \\
& \forall\left(a^{\#}, q_{1}\right), F_{\sigma_{1}}\left[q_{1}\right]\left(a^{\#}, q_{0}\right) \Rightarrow a^{\#} \in \alpha_{\sigma_{1} \circ \sigma_{2}}(E) \\
& \wedge \llbracket c t x\left(\operatorname{vars}^{\prime}\right) \rrbracket_{\mathcal{M}}=\llbracket c t x\left(\operatorname{vars}_{t}\right) \rrbracket_{\mathcal{M}}
\end{aligned}
$$

23) Applying the definition of $F_{\sigma_{1} \circ \sigma_{2}}$ in the reverse way as in step 16

$\forall\left(a^{\#}, q\right)$,

$$
\begin{aligned}
F_{\sigma_{1} \circ \sigma_{2}}[q]\left(a^{\#}, a\left(\operatorname{vars}^{\prime}\right)\right) \Rightarrow a^{\#} & \in \alpha_{\sigma_{1} \circ \sigma_{2}}(E) \\
& \wedge \llbracket c t x(\operatorname{var} s) \rrbracket_{\mathcal{M}}=\llbracket c t x\left(\operatorname{vars}^{\prime}\right) \rrbracket_{\mathcal{M}}
\end{aligned}
$$


24) Our desired result !

25) Let us show Handled $(\emptyset) \Rightarrow \operatorname{Handled}\left(I_{2}\right)$ where the property $\operatorname{Handled}\left(I_{t m p}\right)$ is defined in step 17 To do so, we prove that we have $\operatorname{Handled}(I)$ for any set $I$ reached by the variable $I_{t m p}$ of Algorithm 4 and prove that $\operatorname{Handled}(I) \Rightarrow \operatorname{Handled}\left(I \cup\left\{\left(q_{0}, q_{2}\right)\right\}\right)$ where $I$ and $I \cup\left\{\left(q_{0}, q_{2}\right)\right\}$ are values reached by $I_{t m p}$ in the while loop. It follows by transitivity and finiteness of $I_{2}$ that $\operatorname{Handled}(\emptyset) \Rightarrow \operatorname{Handled}\left(I_{2}\right)$.

The assumption Handled $(I)$ after introducing vars' yields :

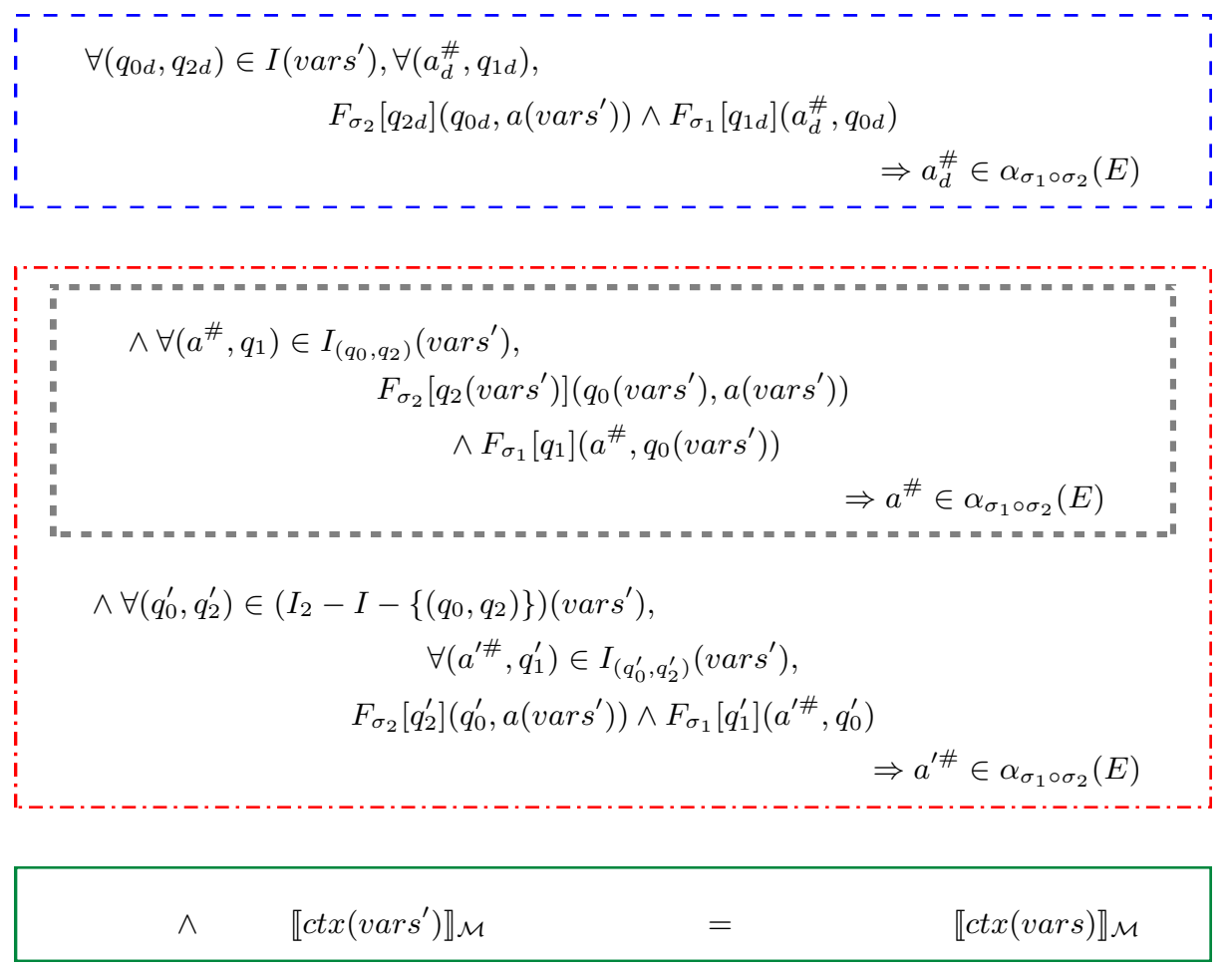

26) Our goal is to "move" the part in gray from within the part in red into the part in blue. Let us work on the part in gray for now.

$$
\begin{aligned}
& \forall\left(a^{\#}, q_{1}\right) \in I_{\left(q_{0}, q_{2}\right)}\left(\text { vars }^{\prime}\right), \\
& \quad F_{\sigma_{2}}\left[q_{2}\left(\text { vars }^{\prime}\right)\right]\left(q_{0}\left(\text { vars }^{\prime}\right), a_{\left.\left(\text {vars }^{\prime}\right)\right) \wedge F_{\sigma_{1}}\left[q_{1}\right]\left(a^{\#}, q_{0}\left(\text { vars }^{\prime}\right)\right)}\right. \\
& \quad \Rightarrow a^{\#} \in \alpha_{\sigma_{1} \circ \sigma_{2}}(E)
\end{aligned}
$$

27) Let us prepare to apply strong completeness of $I_{\left(q_{0}, q_{2}\right)}$ which is equal to $\operatorname{insts}\left(F_{\sigma_{1}}, q_{0},\left(I_{2}-\left\{\left(q_{0}, q_{2}\right)\right\}, I_{f}, F_{\sigma_{2}}\left[q_{2}\right]\left(q_{0}, a\right)\right.\right.$, ctx $\left.)\right)$

$$
\begin{aligned}
& F_{\sigma_{2}}\left[q_{2}\left(\text { vars }^{\prime}\right)\right]\left(q_{0}\left(\text { vars }^{\prime}\right), a\left(\text { vars }^{\prime}\right)\right) \\
& \quad \Rightarrow \forall\left(a^{\#}, q_{1}\right) \in I_{\left(q_{0}, q_{2}\right)}\left(\operatorname{vars}^{\prime}\right), F_{\sigma_{1}}\left[q_{1}\right]\left(a^{\#}, q_{0}\left(\text { vars }^{\prime}\right)\right) \\
& \Rightarrow a^{\#} \in \alpha_{\sigma_{1} \circ \sigma_{2}}(E)
\end{aligned}
$$


28) Let us put $F_{\sigma_{2}}\left[q_{2}\left(\right.\right.$ vars $\left.\left.^{\prime}\right)\right]\left(q_{0}\left(\right.\right.$ vars $\left.^{\prime}\right)$, a $\left(\right.$ vars $\left.\left.^{\prime}\right)\right)$ into context

$$
\left.\forall\left(a^{\#}, q_{1}\right) \in I_{\left(q_{0}, q_{2}\right)}\left(\text { vars }^{\prime}\right), F_{\sigma_{1}}\left[q_{1}\right]\left(a^{\#}, q_{0} \text { vars }^{\prime}\right)\right) \quad \Rightarrow a^{\#} \in \alpha_{\sigma_{1} \circ \sigma_{2}}(E)
$$

29)Let use strong completeness of $I_{\left(q_{0}, q_{2}\right)}$ with $E=\alpha_{\sigma_{2}}(E)$. We remind that $I_{\left(q_{0}, q_{2}\right)}=\operatorname{insts}\left(F_{\sigma_{1}}, q_{0},\left(I_{2}-\left\{\left(q_{0}, q_{2}\right)\right\}, I_{f}, F_{\sigma_{2}}\left[q_{2}\right]\left(q_{0}, a\right), c t x\right)\right)$ with $I_{f}=\left(I_{2}-I-\right.$ $\left.\left\{\left(q_{0}, q_{2}\right)\right\}\right)$. We retrieve $\operatorname{vars}_{t}^{\prime}$ such that

$$
\forall\left(a^{\#}, q_{1}\right), F_{\sigma_{1}}\left[q_{1}\right]\left(a^{\#}, q_{0}\left(\operatorname{vars}_{t}^{\prime}\right)\right) \quad \Rightarrow \quad a^{\#} \quad \in \quad \alpha_{\sigma_{1} \circ \sigma_{2}}(E)
$$$$
\wedge\left(I_{2}-\left\{\left(q_{0}, q_{2}\right)\right\}\right)\left(\operatorname{vars}^{\prime}\right)=\left(I_{2}-\left\{\left(q_{0}, q_{2}\right)\right\}\right)\left(\operatorname{vars}_{t}^{\prime}\right)
$$$$
\wedge \forall\left(q_{0}^{\prime}, q_{2}^{\prime}\right) \in\left(I_{2}-I-\left\{\left(q_{0}, q_{2}\right)\right\}\right)\left(\text { vars }^{\prime}\right),
$$

$$
I_{\left(q_{0}^{\prime}, q_{2}^{\prime}\right)}\left(\operatorname{vars}^{\prime}\right)=I_{\left(q_{0}^{\prime}, q_{2}^{\prime}\right)}\left(\operatorname{vars}_{t}^{\prime}\right)
$$

$\wedge F_{\sigma_{2}}\left[q_{2}\left(\operatorname{vars}^{\prime}\right)\right]\left(q_{0}\left(\right.\right.$ vars $\left.^{\prime}\right), a\left(\right.$ vars $\left.\left.^{\prime}\right)\right)=$

$\wedge \llbracket \operatorname{ctx}\left(\operatorname{vars}^{\prime}\right) \rrbracket_{\mathcal{M}}=\llbracket \operatorname{ctx}\left(\operatorname{vars}_{t}^{\prime}\right) \rrbracket_{\mathcal{M}}$

$$
F_{\sigma_{2}}\left[q_{2}\left(\operatorname{var}_{t}^{\prime}\right)\right]\left(q_{0}\left(\operatorname{vars}_{t}^{\prime}\right), a\left(\operatorname{var}_{t}^{\prime}\right)\right)
$$

30) Let us use the equalities and set ourselves in the context of steps 26 and 28

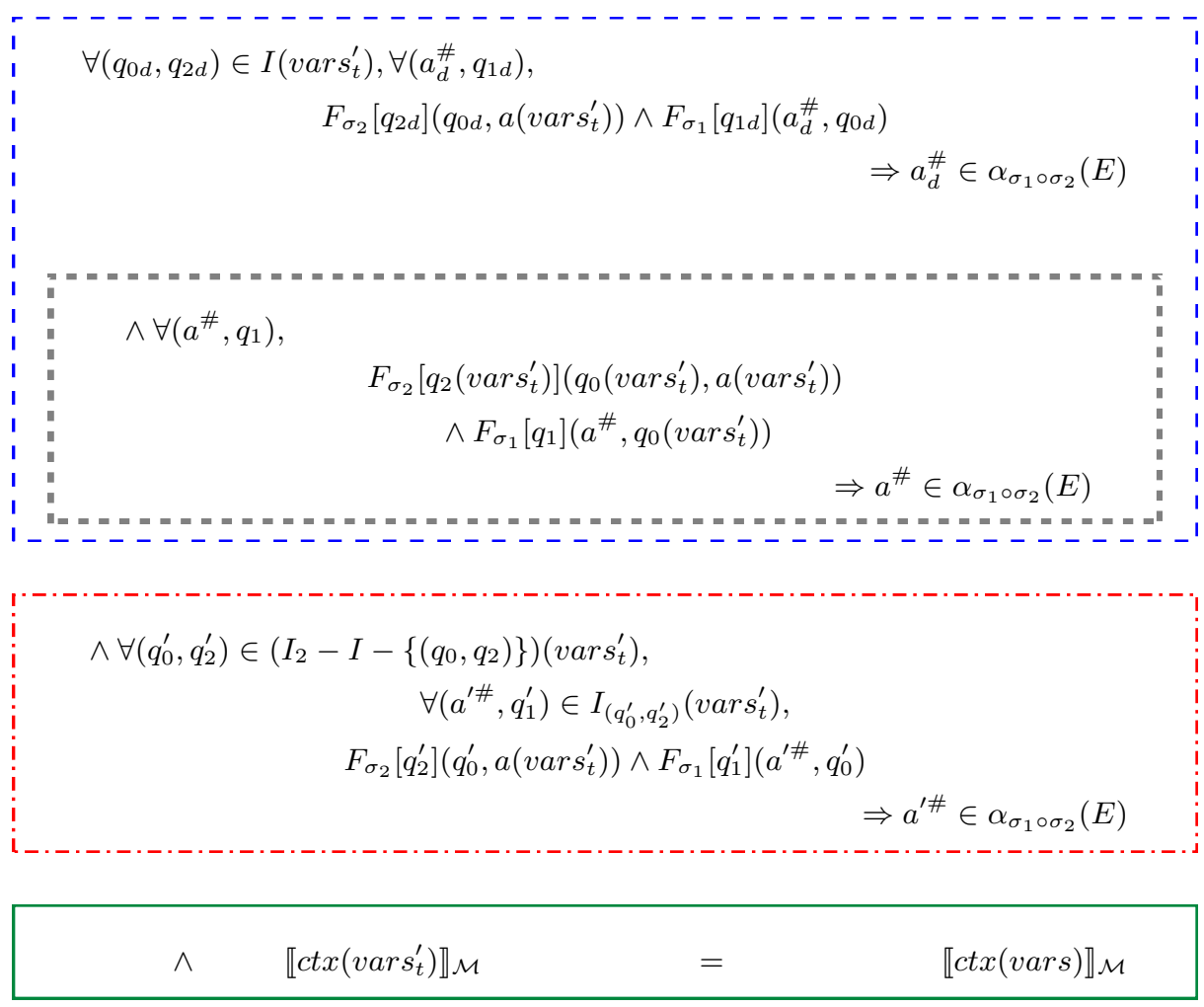

31) Handled $\left(I \cup\left\{\left(q_{0}, q_{2}\right)\right\}\right)$, our desired result ! 


\section{A.3 Cell abstraction results}

Theorem 10 (Abstraction compatibility for cell abstractions). The $a b$ stractions of Example 9 have strongly complete instantiation heuristics when $\mathrm{Cell}_{n}$ has.

Proof. As the abstractions are $\mathrm{Cell}_{n}$ are finite abstractions, the only consideration is compatibility of the abstractions (condition for $\circ$ to have strongly complete instantiation). Let us prove the result for $\mathrm{Cell}_{1}$ and consider the composed abstraction $\sigma_{1}$. We need to prove $: \forall E \neq \emptyset, \alpha_{C_{e l l}} \circ \gamma_{C_{e l l}} \circ \gamma_{\sigma_{1}} \circ \alpha_{\sigma_{1}} \circ \alpha_{C e l l_{1}}(E)=$ $\gamma_{\sigma_{1}} \circ \alpha_{\sigma_{1}} \circ \alpha_{C_{\text {Cell }}}(E)$.

Introduce $E \neq \emptyset$ and define $X=\gamma_{\sigma_{1}} \circ \alpha_{\sigma_{1}} \circ \alpha_{C e l l_{1}}(E)$. Note that $\exists a, \forall i,(i, a[i]) \in$ $X$ because $E \neq \emptyset$, thus, $\exists a \in E$, thus, $\exists a, \forall i,(i, a[i]) \in \alpha_{C_{\text {ell }}}(E)$, thus, $\exists a, \forall i,(i, a[i]) \in$ $X$ as $\forall S, S \subseteq \gamma_{\sigma_{1}} \circ \alpha_{\sigma_{1}}(S)$.

We now prove $\alpha_{\text {Cell }_{1}} \circ \gamma_{\text {Cell }_{1}}(X)=X$.

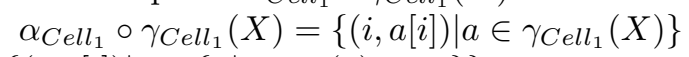

$=\left\{(i, a[i]) \mid a \in\left\{a \mid \sigma_{\text {Cell }_{1}}(a) \subseteq X\right\}\right\}$

$=\{(i, v) \mid \exists a \in\{a \mid \sigma(a) \subseteq X\} \wedge v=a[i]\}$

$=\left\{(i, v) \mid \exists a, \sigma_{\text {Cell }_{1}}(a) \subseteq X \wedge v=a[i]\right\}$

$=\{(i, v) \mid \exists a, \forall i,(i, a[i]) \in X \wedge v=a[i]\}$

$=\{(i, v) \mid$ true $\wedge(i, v) \in X\}$ as proved above

$=X$

Theorem 11 (Correctness of relevant $(a$, expr $))$. If $\top \notin \operatorname{relevant}(a$, expr $)$ then

$$
\begin{aligned}
\forall \mathcal{M}, \operatorname{vars}, a^{\prime},(\forall i & \left.\in \operatorname{relevant}(a, \operatorname{expr}), a^{\prime}[i]=a(\operatorname{vars})[i]\right) \Rightarrow \\
& \exists \operatorname{vars^{\prime }}, \llbracket \operatorname{expr}(\operatorname{vars}) \rrbracket_{\mathcal{M}}=\llbracket \operatorname{expr}\left(\operatorname{vars}^{\prime}\right) \rrbracket_{\mathcal{M}} \wedge a\left(\operatorname{vars}^{\prime}\right)=a^{\prime}
\end{aligned}
$$

Remark 11. Should the relevant algorithm be expanded, one only needs to keep the property of this theorem to have strong completeness of Cell ${ }_{n}$.

Proof. Let us first prove a property about read : if read(avar,expr) does not contain $T$, then

$$
\begin{aligned}
& \forall \mathcal{M}, a, a^{\prime},\left(\forall i \in \operatorname{read}(\text { avar }, \text { expr }), a[i]=a^{\prime}[i]\right) \Rightarrow \\
& \forall \text { vars, } \llbracket \operatorname{expr}\left(\operatorname{vars}[\text { avar } \leftarrow a] \rrbracket_{\mathcal{M}}=\llbracket \operatorname{expr}\left(\operatorname{vars}\left[\text { avar } \leftarrow a^{\prime}\right] \rrbracket_{\mathcal{M}}\right.\right.
\end{aligned}
$$

This proof is done by induction on expr. Now, let us introduce $\mathcal{M}$, vars,$a^{\prime}$ and

let us assume $\forall i \in \operatorname{relevant}(a, \operatorname{expr}), a^{\prime}[i]=a($ vars $)[i]$

Because $\top \notin$ relevant (a, expr), introduce avar such that $a=$ arrayStoreChain(avar, $I, V)$.

We can use our property on read with $\mathcal{M}=\mathcal{M}, a=a($ vars $), a^{\prime}=a^{\prime}$, vars $=$ vars as:

$\forall i \in \operatorname{read}($ avar, expr $), a($ vars $)[i]=a^{\prime}[i] .($ In fact $\operatorname{read}($ avar, expr $) \subseteq \operatorname{relevant}(a$, expr $)$ 
and $\forall i \in \operatorname{relevant}(a$, expr $), a^{\prime}[i]=a($ vars $\left.)[i]\right)$. Therefore, we have :

$\llbracket \operatorname{expr}(\operatorname{vars}[$ avar $\leftarrow a($ vars $)]) \rrbracket_{\mathcal{M}}=\llbracket \operatorname{expr}\left(\operatorname{vars}\left[\right.\right.$ avar $\left.\left.\leftarrow a^{\prime}\right]\right) \rrbracket_{\mathcal{M}}$

$\equiv \llbracket \operatorname{expr}(\operatorname{vars}) \rrbracket_{\mathcal{M}}=\llbracket \operatorname{expr}\left(\operatorname{vars}\left[\right.\right.$ avar $\left.\left.\leftarrow a^{\prime}\right]\right) \rrbracket_{\mathcal{M}}$

$\equiv \llbracket \operatorname{expr}(\operatorname{vars}) \rrbracket_{\mathcal{M}}=\llbracket \operatorname{expr}\left(\operatorname{vars}^{\prime}\right) \rrbracket_{\mathcal{M}}$

with vars $^{\prime}=$ vars $\left[\right.$ avar $\left.\leftarrow a^{\prime}\right]$, which verifies $a\left(\right.$ vars $\left.^{\prime}\right)=a^{\prime}$

\section{Theorem 12 (Strong Completeness for cell Abstraction).}

Any call to insts $\left(F_{\sigma_{\text {Cell }_{n}}}, a, c t x\right)$ is strongly complete whenever $\top \notin \operatorname{relevant}(a, c t x)$.

Proof. We shall refer to Ind, I etc. as defined in Algorithm. 6. For simplicity, let us write everything as though

insts $\left(F_{\sigma_{\text {Cell }_{n}}}, a, c t x\right)$ returned $I^{n}$ instead of $\left(I^{n},()\right)$. Introduce vars, $E, \mathcal{M}$ and assume

$$
\forall a^{\#} \in \operatorname{insts}\left(F_{\sigma_{\text {Cell }_{n}}}, a, c t x\right)(\text { vars }), F_{\sigma_{\text {Cell }_{n}}}\left(a^{\#}, a(\text { vars })\right) \Rightarrow a^{\#} \in \alpha_{\sigma_{\text {Celln }_{n}}}(E)
$$

which is equivalent to

$$
\operatorname{insts}\left(F_{\sigma_{\mathrm{Cell}_{n}}}, a, c t x\right)(\text { vars }) \cap \sigma_{\mathrm{Cell}_{n}}(\text { (a }(\text { vars })) \subseteq \alpha_{\sigma_{\mathrm{Cell}_{n}}}(E)
$$

which is equivalent to (as $\mathrm{Cell}_{n}$ is just $n$ replication of $C e l l_{1}$ )

$$
\operatorname{insts}\left(F_{\sigma_{\text {Cell }_{1}}}, a, \text { ctx }\right)(\text { vars }) \cap \sigma_{\text {Cell }_{1}}(\text { (a }(\text { vars })) \subseteq \alpha_{\sigma_{\text {Cell }_{1}}}(E)
$$

We need to show (using the same simplifications) :

$$
\exists \operatorname{vars}^{\prime}, \llbracket c t x(\text { vars }) \rrbracket_{\mathcal{M}}=\llbracket c t x\left(\text { vars }^{\prime}\right) \rrbracket_{\mathcal{M}} \wedge \sigma_{\text {Cell }_{1}}\left(a\left(\text { vars }^{\prime}\right)\right) \subseteq \alpha_{\sigma_{\text {Cell }_{1}}}(E)
$$

1. Case $E=\emptyset$ :

Let us prove that the assumption 15 is not verified and thus by absurdum, our result stands.

To prove the assumption if not verified, we use :

(a) $\alpha_{\sigma_{\text {Cell }_{1}}}(E)=\alpha_{\sigma_{\text {Cell }_{1}}}(\emptyset)=\emptyset$

(b) insts $\left(F_{\sigma_{\text {Cell }_{1}}}, a\right.$, ctx $)($ vars $) \cap \sigma_{\text {Cell }_{1}}($ (a (vars $\left.)\right) \neq \emptyset$ As insts $\left(F_{\sigma_{\text {Cell }_{1}}}, a\right.$, ctx $)($ vars $) \neq$

$\emptyset$ by construction of Ind and insts $\left(F_{\sigma_{\text {Cell }}}, a\right.$, ctx $)$ (vars $) \subseteq \sigma_{\text {Cell }_{1}}($ a (vars $\left.)\right)$

by construction of $I$ (we chose $(a, a[i])$ ).

2. Continuing with $E \neq \emptyset$

Let $a_{\top} \in E$

3. Let $a^{\prime}$ such that

$\forall i, a^{\prime}[i]=i t e\left(i \in \operatorname{relevant}(a, c t x), a(\right.$ vars $\left.)[i], a_{\top}[i]\right)$

4. By assumption $\top \notin \operatorname{relevant}(a, \operatorname{ct} x)$ and by construction $\forall i \in \operatorname{relevant}(a, c t x), a^{\prime}[i]=$ a(vars $)[i]$, therefore

$\exists$ vars $^{\prime}, \llbracket c t x($ vars $) \rrbracket_{\mathcal{M}}=\llbracket c t x\left(\right.$ vars $\left.^{\prime}\right) \rrbracket_{\mathcal{M}} \wedge a\left(\right.$ vars $\left.^{\prime}\right)=a_{\top}$ using Theorem 11

5. We already have $\llbracket \operatorname{ctx}($ vars $) \rrbracket_{\mathcal{M}}=\llbracket \operatorname{ctx}\left(\right.$ vars $\left.^{\prime}\right) \rrbracket_{\mathcal{M}}$. Thus, we only need

$$
\sigma_{\text {Cell }_{1}}\left(a\left(\text { vars }^{\prime}\right)\right) \subseteq \alpha_{\sigma_{\text {Cell }_{1}}}(E)
$$

Which is equivalent to

$$
\left\{\left(i, a^{\prime}[i]\right)\right\} \subseteq \alpha_{\sigma_{\text {Cell }_{1}}}(E)
$$

Which is verified as 
(a) $\left\{\left(i, a^{\prime}[i]\right), i \in \operatorname{relevant}(a, c t x)\right\}=\{(i, a($ vars $)[i]), i \in \operatorname{relevant}(a, \operatorname{ct} x)\} \subseteq$ insts $\left(F_{\sigma_{\text {Cell }_{1}}}, a, c t x\right)$ (vars) which by assumption is included in $\alpha_{\sigma_{\text {Cell }_{1}}}(E)$

(b) $\left\{\left(i, a^{\prime}[i]\right), i \notin \operatorname{relevant}(a\right.$, ctx $\left.)\right\}$

$=\left\{\left(i, a_{\top}[i]\right), i \notin \operatorname{relevant}(a, c t x)\right\}$

$\subseteq \sigma_{\text {Cell }_{1}}\left(a_{\top}\right) \subseteq \alpha_{\text {Cell }_{1}}\left(\left\{a_{\top}\right\}\right) \subseteq \alpha_{C^{\prime} e l l_{1}}(\{E\})$

as $a_{\top} \in E$.

Theorem 14 (Satisfiability preservation of trs). If the input clauses are normalized Horn clauses such that there is at most one negative predicate in each clause (corresponds to program without function calls) and the theory on which arrays are used contains read, write but no array equalities $a=a^{\prime}$ and there are no arrays whose index or value type is an array and no array variable appears twice within the same predicate then:

$$
\operatorname{trs}(\mathfrak{C}, n) \text { satisfiable } \equiv \exists \mathcal{M} \text { expressible by } \mathcal{G}, H_{C}(\mathcal{M})
$$

Where $\mathcal{G}$ is the abstraction used, that is, $\mathcal{G}_{\sigma^{P_{1}}}^{P_{1}} \circ \ldots \circ \mathcal{G}_{\sigma_{m}}^{P_{m}}$ with $P_{1}, \ldots, P_{m}$ are the predicates of $\mathfrak{C}$ and $\sigma^{P_{i}}$ is as defined in Algorithm 7 and $\mathcal{G}_{\sigma}^{P}$ is as defined in Definition 8.

Proof. First, note that $\mathcal{G}$ does not use "data abstraction composition combinator", and can be defined by $\alpha_{\mathcal{G}}(\mathcal{M})\left(P_{i}\right)=\alpha_{\sigma^{P_{i}}}\left(\mathcal{M}\left(P_{i}\right)\right)$. As all our other combinators are strongly complete when their parameters are, we only need to prove that all calls to insts $\left(\mathrm{Cell}_{n}, a, c t x\right)$ are strongly complete, which is equivalent to $\top \notin \operatorname{relevant}(a, \operatorname{ct} x)$.

We thus need to examine the cases for relevant $(a, c t x)$ as defined in Algorithm 5

1. An array store chain of $a$ (named $\operatorname{asc}(a)$ ) is only used in a read. This is equivalent to stating that $a s c(a)$ is not used in (i) array equalities (ii) nor within predicates of the context, (iii) nor in a tuple constructor. (i) is true since initial clauses do not contain array equalities initially and we do not introduce any. As all arrays of all predicates are abstracted, predicates do not contain array typed parameters (ii). Only the dot combinator introduce tuples constructors, and no array variable appears twice within the same predicate (iii).

2. ctx does not contain quantifiers. Initially, there are no quantifiers, and the quantifiers introduced by the abstractions are either positive predicates, and thus are transformed into free variables; or a unique negative predicate which is instanciated just afterwards. Thus, there are no quantifiers in the context. 